\title{
EFFICIENCY OF NEARLY PERIODIC STRUCTURES FOR MITIGATION OF GROUND VIBRATION
}

\author{
Lars V. Andersen ${ }^{1}$, Andrew Peplow ${ }^{2}$, and P. Bucinskas ${ }^{1}$ \\ ${ }^{1}$ Department of Civil Engineering, Aalborg University \\ Thomas Manns Vej 23, DK-9220 Aalborg East, Denmark \\ e-mail: \{la,pbu\}@civil.aau.dk \\ ${ }^{2}$ Department of Natural Sciences and Public Health, CSSH, Zayed University \\ P.O. Box 144534, Abu Dhabi, United Arabic Emirates \\ Andrew.Peplow@zu.ac.ae
}

Keywords: Wave propagation, wave-impeding block, WIB, layered soil, insertion loss.

\begin{abstract}
Periodic structures are known to produce passbands and stopbands for propagation of vibration energy within the frequency domain. Sources vibrating harmonically at a frequency within a passband can lead to propagation of energy through propagating modes over long distances. However, sources vibrating at a frequency within a stopband excite only nearfields in the form of attenuating and evanescent modes, and the energy decays with distance. The decay phenomena are due to destructive interference of waves reflected and scattered by interfaces or obstacles placed periodically within or between the repeated cells of the structure. For a truly periodic structure, the vibration level within a stopband goes toward zero after infinitely many repetitions of the cell. For example, employing a two-dimensional model, Andersen [1] found that stopbands for ground vibration in the low-frequency range can be introduced by periodic inclusions or changes to the ground surface geometry.
\end{abstract}

However, for vibration mitigation in the context of real civil-engineering problems related to ground-borne noise from railways, for example, the excitation is not strictly harmonic and a steady state of the response is usually not achieved. Further, only a limited number of repetitions of wave impedance blocks or barriers can be made in practice, and in three dimensions, the inclusions have finite extent in the direction orthogonal to the array. Similarly to the work by Andersen et al. [2], this paper addresses the question whether repeated structures of nearly periodic nature can be used to mitigate vibrations caused by non-stationary sources. For this purpose, wave impedance blocks with finite numbers of repetitions are compared to their truly periodic counterparts. Firstly, a two-dimensional study is conducted with focus on studying the nature of wave modes in a periodic array of wave impeding blocks. Secondly, three-dimensional analysis is performed in the frequency domain, focusing on the insertion loss provided by increasing numbers of repetitions of blocks with different height and embedment. Finally, the insertion loss provided by nearly periodic structures is examined, and the mitigation efficiency of wave-impeding-block arrays is quantified in the case of transient loads. 


\section{INTRODUCTION}

Ground vibration from traffic, construction work and rotating machinery cause annoyance to people living near railways, roads, construction sites, factories and workshops. Therefore, many researchers have proposed mitigation of vibration by various methods, including trenches, barriers and wave-impeding blocks (WIBs). Barriers can be made of materials that are either soft and light or stiff and heavy relatively to the soil. The governing principle is in any case to produce a mechanical-impedance mismatch between the barrier material and the soil, leading to reflection rather than transmission of energy. A wave-impeding block can be placed on the ground surface in which case it mainly contributes by its mass, or it can be embedded in the ground, adding stiffness to the soil.

Warburton et al. [3] performed one of the earliest studies of the harmonic response of a single heavy mass placed on the surface of an elastic half-space. The efficiency of such masses for mitigation of harmonic vibration from traffic was studied by Krylov [4]. Recently, Dijckmans et al. [5] introduced the idea of placing an array of heavy masses along a railway track in order to mitigate vibration. The solution was found to have a significant effect.

Peplow et al. [6] introduced the idea of embedding a rigid block in the soil below a source in order to mitigate ground vibration by stiffening the soil. This so-called wave-impeding block (WIB) was found to have a significant impact on wave propagation in a layered ground, depending on the properties of the soil. More recently, a similar study was performed by Peplow and Finnveden [7], but this time based on a wave-based finite-element formulation. Takemiya [8] studied WIBs formed by piles placed in a honeycomb pattern around the foundation for a pylon supporting a railway bridge. The chosen WIB geometry was found advantageous in terms of mitigating waves in the considered frequency range. Along the same line, Masoumi et al. [9] demonstrated the efficiency of wave-impeding blocks regarding the mitigation of ground vibration from railway traffic. They applied a numerical model validated by experimental work.

Andersen and Nielsen [10] conducted a study of ground vibration from a moving force on a railway track, employing a coupled finite-element/boundary-element (FE/BE) model. They analysed various vibration mitigation methods, including soil stiffening under the rail and trenches or barriers along the track. Andersen and Liingaard [11] performed a detailed study of vibration barriers formed by one or more sheet pile walls. Especially, the influence of barrier position and inclination was analysed using a two-dimensional FE/BE model. Their work was followed up by Andersen and Augustesen [12] who used a three-dimensional FE/BE model to study the efficiency of inclined, embedded barriers placed along a railway. They concluded that inclined barriers might perform better than vertical barriers when a concrete lid needs to be placed on top to prevent people or water from entering the trench. A further study of optimization of wave barriers was performed by van Hoorickx et al. [13].

Instead of relying on the screening effect of a single barrier or the scattering provided by a single WIB, the possibly destructive interference caused by repeated inclusions can be utilized. Mead [14] studied the effect of periodicity in a structure, finding that bandgaps occur in certain frequency ranges depending on the mechanical properties of the materials and the geometry of the cell which is repeated along the periodic array. Based on this idea, Andersen [1] proposed the inclusion of periodic structures in the ground to mitigate vibration. Using a two-dimensional FE model and Floquet theory, he demonstrated that stopbands are produced by arrays of WIBs or ground surface changes in the form of small hills and valleys repeated in a periodic manner. Persson et al. [15] also studied the concept of ground-surface landscaping to mitigate vibration. Finally, Andersen et al. [2] conducted a number of small-scale experiments and FE analyses with the aim to quantify the insertion loss provided by placing one or more blocks on or in the ground within the transmission path between a source and a receiver. 
The present paper follows the idea of placing an array of rigid blocks within the propagation path between a source and a receiver point located on the ground surface. Section 2 presents the methodology applied for analysis of wave propagation in the layered soil. Two approaches are described: a two-dimensional (2D) model of a truly periodic array of wave impeding blocks (WIBs), and a three-dimensional (3D) model of a finite WIB array. The results of the 2D model are presented in Section 3 with focus on studying mitigation efficiency of the WIB array, whereas Section 4 presents the results of analyses performed with the 3D model for stationary and transient loads. Finally, the main conclusions are given in Section 5.

\section{METHODOLOGY}

Wave propagation in soil with two layers overlying bedrock has been analysed by two methods, especially regarding the efficiency of an array of WIBs in mitigating ground vibration. The first model is 2D, assuming the WIB array to be infinite and with each block having an infinite extent in the direction out of the plane. Here a periodic model based on Floquet transformation has been applied with the purpose of identifying passbands and stopbands for travelling waves, and furthermore a quantification has been made regarding the number of attenuating and evanescent modes that have a decay rate with distance below a certain threshold. This leads to a quantification of the number of modes that are present in the plane-wave-propagation problem with various configurations of the WIB array, and to a qualitative assessment of the mitigation efficiency. The second model is three-dimensional (3D) and employs Green's function for wave propagation in a stratum. This model has been utilized for analysis of the insertion loss (IL) provided by inclusion of arrays with few or more WIBs placed with constant or nearly constant centre-to-centre distance, providing a nearly periodic array. Whereas all other studies have been done in the frequency domain, a final study is reported in which the transient response to a pulse load has been analysed in order to assess the effects of WIBs for different numbers of blocks.

\subsection{Finite-element model of a periodic structure}

The starting point for analysis of wave propagation in the periodic 2D layered soil-WIB system is the stiffness matrix $\mathbf{K}$ and the mass matrix $\mathbf{M}$ for a reference cell. With reference to Figure 1, Cell 0 has been modelled, and the effect of having infinitely many repetitions of cells along the WIB array has been obtained by boundary conditions based on Floquet periodicity. In frequency domain, this leads to a set of equations that can be solved in order to determine the number of propagating, attenuated and evanescent wave modes in the periodic system.

Two possibilities exist: 1) Real-valued discrete wavenumbers, $k$, are defined, and for each value of $k$, a first-order eigenvalue problem is obtained in terms of the angular frequency $\omega$; 2) discrete values of $\omega$ are defined, leading to a second-order eigenvalue problem in terms of $k$. Approach number 1 is only useful to identify propagating wave modes, whereas the second approach identifies all wave modes, i.e. propagating as well as attenuated or evanescent modes. Approach number 2 is therefore considered here.

Introducing $\mathbf{U}_{\mathrm{b}}$ and $\mathbf{F}_{\mathrm{b}}$ as the reduced vectors storing the nodal degrees of freedom and nodal forces, respectively, at the left (L) and right (R) boundaries of the reference cell, the governing equation for wave propagation in the FE model can be expressed as:

$$
\widetilde{\mathbf{D}} \mathbf{U}_{\mathrm{b}}=\mathbf{F}_{\mathrm{b}}, \quad \widetilde{\mathbf{D}}=\left[\begin{array}{cc}
\widetilde{\mathbf{D}}_{\mathrm{LL}} & \widetilde{\mathbf{D}}_{\mathrm{LR}} \\
\widetilde{\mathbf{D}}_{\mathrm{RL}} & \widetilde{\mathbf{D}}_{\mathrm{RR}}
\end{array}\right], \quad \mathbf{U}_{\mathrm{b}}=\left\{\begin{array}{l}
\mathbf{U}_{\mathrm{L}} \\
\mathbf{U}_{\mathrm{R}}
\end{array}\right\}, \quad \mathbf{F}_{\mathrm{b}}=\left\{\begin{array}{l}
\mathbf{F}_{\mathrm{L}} \\
\mathbf{F}_{\mathrm{R}}
\end{array}\right\},
$$

where $\widetilde{\mathbf{D}}$ is the reduced dynamic stiffness matrix obtained by condensing the full dynamic stiffness matrix $\mathbf{D}=\mathbf{K}-\omega^{2} \mathbf{M}$ which accounts for all degrees of freedom at all nodes on the boundary of the cell as well as inside the cell. It should be noted that all quantities in Eq. (1) depend 
on $\omega$, i.e. $\mathbf{U}_{\mathrm{b}}=\mathbf{U}_{\mathrm{b}}(\omega)$, etc., and that the vectors belonging to the left and right boundaries of the cell have equal length, given that the nodes must be placed at the same vertical position to allow a periodic coupling in the format

$$
\mathbf{U}_{\mathrm{R}}=\exp (-\mathrm{i} k \Delta) \mathbf{U}_{\mathrm{L}}=\lambda \mathbf{U}_{\mathrm{L}}, \quad \exp (-\mathrm{i} k \Delta) \mathbf{F}_{\mathrm{L}}+\mathbf{F}_{\mathrm{R}}=\lambda \mathbf{F}_{\mathrm{L}}+\mathbf{F}_{\mathrm{R}}=\mathbf{0}_{\mathrm{L}} .
$$

Here $\mathrm{i}=\sqrt{-1}, k$ is the wavenumber, $\Delta=w_{1}$ is the width of the cell (see Figure 1), and $\lambda$ is the so-called propagation constant which is in general complex. Propagating modes are identified by having real-valued wavenumbers and a propagation constants defined by $|\lambda|=1$.

In the present analyses, the commercial finite-element (FE) code ABAQUS 6.14 [16] has been employed to calculate the matrices $\mathbf{K}$ and $\mathbf{M}$ for reference cells with different configurations of the WIB. Details regarding material properties are given in Sections 2.4 and 3. Assuming plane strain, quadrilateral elements with eight nodes and quadratic interpolation of the displacement field are employed, using reduced integration of the stresses. A mesh size of $1.0 \mathrm{~m}$ has been used everywhere in the plane FE model, which provides an accurate discretization of the waves within the frequency range $0-25 \mathrm{~Hz}$. The remaining part of the analysis, i.e. introduction of the periodicity conditions (2) and solution of the eigenvalue problem in terms of the propagation constant $\lambda$, has been performed in Matlab.

As a note, the wave propagation pattern related to a dynamic load applied over a finite area on the surface of the ground is in essence three-dimensional. Hence, the obvious choice for analysis of mitigation related to a periodic array of WIBs would be a 3D FE model. However, when periodicity only occurs in one direction, namely in the direction of the WIB array, the periodicity conditions should only be implemented at the "ends" of the reference cell, whereas transmitting boundary conditions should be applied on the "sides" of the 3D reference cell. This may be in the form of, for example, dashpots providing low reflection as suggested by Lysmer and Kuhlemeyer [17], or by implementation of Perfectly Matched Layers (PMLs) as suggested by Chew and Liu [18]. However, such absorbing boundary conditions provide dissipation equivalent of geometrical dissipation in an unbounded domain. Therefore, all waves in a 3D model of a one-dimensional WIB array become attenuated, making it difficult to assess the efficiency of the WIBs. Hence, the 2D model has been utilized in this paper.

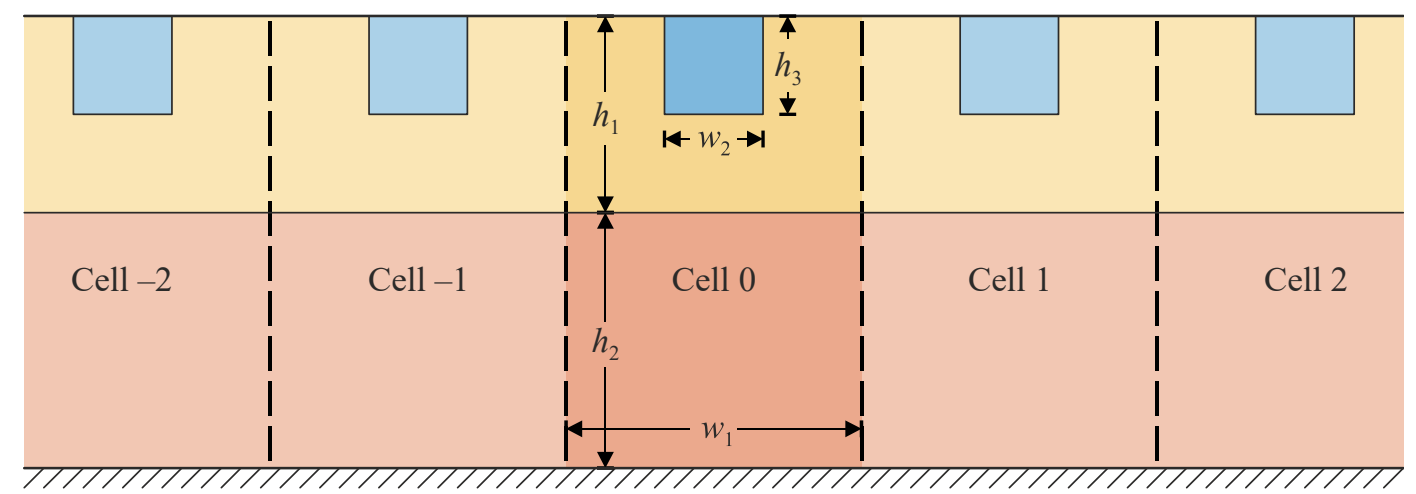

Figure 1: Periodic placement of WIBs into layered soil.

\subsection{Semi-analytical model based on Green's function for a stratum}

Consider a viscoelastic stratum overlying bedrock. All interfaces as well as the fixed base and free surface are horizontal, and the material in each layer is homogeneous. With these simplifications, the displacement $\mathbf{u}(x, y, z, t)$ at the spatial point $(x, y, z)$ and time $t$ are related to the traction $\mathbf{p}\left(x^{\prime}, y^{\prime}, z^{\prime}, t^{\prime}\right)$ applied at the point $\left(x^{\prime}, y^{\prime}, z^{\prime}\right)$ and time $t^{\prime}$ by the Green's function $\mathbf{g}\left(x-x^{\prime}, y-y^{\prime}, z, z^{\prime}, t-t^{\prime}\right)$. Hence, the total response at $(x, y, z)$ can be determined as 


$$
\begin{array}{r}
\mathbf{u}(x, y, z, t)=\int_{-\infty}^{t} \int_{-\infty}^{0} \int_{-\infty}^{\infty} \int_{-\infty}^{\infty} \mathbf{g}\left(x-x^{\prime}, y-y^{\prime}, z, z^{\prime}, t-t^{\prime}\right) \\
\cdot \mathbf{p}\left(x^{\prime}, y^{\prime}, z^{\prime}, t^{\prime}\right) d x^{\prime} d y^{\prime} d z^{\prime} d t^{\prime}
\end{array}
$$

The Green's function for a stratum cannot be found analytically in the space-time domain, and the quadruple convolution over time and space is computationally inefficient. Therefore, the idea is instead to formulate the problem in horizontal wavenumber-frequency domain:

$$
\mathbf{U}\left(k_{x}, k_{y}, z, \omega\right)=\mathbf{G}\left(k_{x}, k_{y}, z, z^{\prime}, \omega\right) \mathbf{P}\left(k_{x}, k_{y}, z^{\prime}, \omega\right) \text {, }
$$

where $\mathbf{U}\left(k_{x}, k_{y}, z, \omega\right), \mathbf{P}\left(k_{x}, k_{y}, z^{\prime}, \omega\right)$, and $\mathbf{G}\left(k_{x}, k_{y}, z, z^{\prime}, \omega\right)$ are the triple Fourier transforms of the displacement, traction and Green's function, respectively. The Green's function for a stratum formulated in horizontal wavenumber-frequency domain can be determined analytically. To do this, a $6 \times 6$ transfer matrices is first established for each soil layer (or sublayer) as proposed by Haskell [19] and Thompson [20]. With reference to Figure 2, the transfer matrix for layer $j$ relates the three displacement components and the three traction components at the top interface $j 0$ to those at the bottom interface $j 1$. Due to continuity of the displacements and equilibrium of the stresses across the interfaces, it follows that a transfer matrix for the entire ground can be found be simple multiplication of the layer transfer matrices. This method was utilized, for example, by Sheng et al. [21] for analysis of railways.

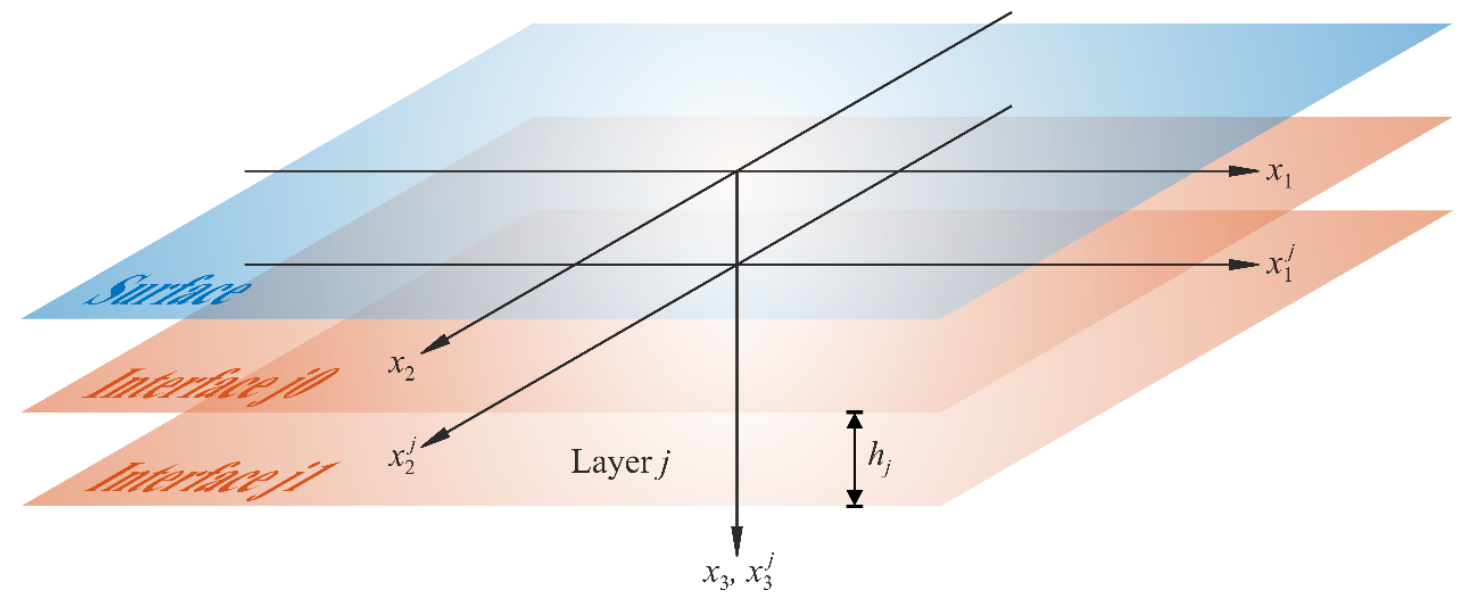

Figure 2: Strata analysed by semi-analytical model.

\subsection{Computation model of wave impedance blocks embedded in a stratum}

Based on the Green's function $\mathbf{G}\left(k_{x}, k_{y}, z, z^{\prime}, \omega\right)$ for layered soil, Andersen and Clausen [22] formulated an efficient method for analysis of rigid footings placed on the surface of the ground. This method has been extended to analysis of rigid bodies embedded in the soil. As suggested by Andersen [23] and Bucinskas et al. [24], structure-soil-structure interaction between $N_{\mathrm{F}}$ rigid bodies can be expressed in terms of the impedance matrix $\mathbf{D}_{\mathrm{F}}(\omega)$,

$$
\mathbf{D}_{\mathrm{F}}(\omega) \mathbf{U}_{\mathrm{F}}(\omega)=\mathbf{F}_{\mathrm{F}}(\omega), \quad \mathbf{U}_{\mathrm{F}}(\omega)=\left\{\begin{array}{c}
\mathbf{U}_{\mathrm{f}, 1}(\omega) \\
\mathbf{U}_{\mathrm{f}, 2}(\omega) \\
\vdots \\
\mathbf{U}_{\mathrm{f}, N_{\mathrm{F}}}(\omega)
\end{array}\right\}, \quad \mathbf{F}_{\mathrm{F}}(\omega)=\left\{\begin{array}{c}
\mathbf{F}_{\mathrm{f}, 1}(\omega) \\
\mathbf{F}_{\mathrm{f}, 2}(\omega) \\
\vdots \\
\mathbf{F}_{\mathrm{f}, N_{\mathrm{F}}}(\omega)
\end{array}\right\},
$$

where $\mathbf{U}_{\mathrm{f}, j}^{T}(\omega)=\left\{U_{x, j} U_{y, j} U_{z, j} \Theta_{x, j} \Theta_{y, j} \Theta_{z, j}\right\}, j=1,2, \ldots, N_{\mathrm{F}}$, are vectors with the six displacement and rotation degrees of freedom of the rigid bodies, and the corresponding forces and moments are stored in the vectors $\mathbf{F}_{\mathrm{f}, j}^{T}(\omega)=\left\{P_{x, j} P_{y, j} P_{z, j} M_{x, j} M_{y, j} M_{z, j}\right\}$. 
To obtain the matrix $\mathbf{D}_{\mathrm{F}}(\omega)$, the rigid bodies are discretized into a number of so-called soilstructure-interaction points. The Green's function provides the influence from one point to itself and every other point, and it can thus be used to derive a flexibility matrix for the system. This matrix is inverted and post multiplied by a matrix with the displacement of associated with each individual rigid-body mode $\mathbf{D}_{\mathrm{F}}(\omega)$. The Green's function can finally be used to calculate displacements at observation points placed, for example, on the surface of the ground.

\subsection{Definition of soil properties and wave-impedance-block arrays}

A stratum with two layers of soil overlying bedrock has been analysed. Material properties and layer depths are listed in Table 1. In the 2D models, the WIBs consist of an elastic material, the properties of which are also listed in the table, whereas the WIBs are considered rigid blocks with the same mass density within the 3D model based on the transfer-matrix method.

The efficiency of a WIB array regarding vibration mitigation and formation of bandgaps in various frequency ranges depends on the distance between individual WIBs as well as the number of WIBs. Andersen [1] studied numerous configurations regarding the width of the reference cell, $w_{1}$, but in the present work the study will be confined to $w_{1}=18 \mathrm{~m}$. Further, in the 3D models, the length of the WIBs has as well been taken as $18 \mathrm{~m}$, and the WIBs all have a width of $w_{2}=2 \mathrm{~m}$. The load is applied on a rigid massless plate with dimensions $18 \times 2 \mathrm{~m}^{2}$ placed on the ground surface. Figure 3 shows example results of the 3D model for a harmonic load applied at the frequency $6 \mathrm{~Hz}$ and for an array consisting of six $2 \mathrm{~m}$ high WIBs placed equidistantly and embedded $2 \mathrm{~m}$ into the ground. The total length of the model is $180 \mathrm{~m}$, and the vertical cut goes $20 \mathrm{~m}$ into the ground. The presence of the layer interface at $10 \mathrm{~m}$ depth is clear.

\begin{tabular}{llllll}
\hline Material & Depth $(\mathrm{m})$ & Young's modulus $(\mathrm{MPa})$ & Poisson's ratio $(-)$ & Mass density $\left(\mathrm{kg} / \mathrm{m}^{3}\right)$ & Loss factor $(-)$ \\
\hline Soft topsoil & 10 & 50 & 0.48 & 2000 & 0.020 \\
Stiff subsoil & 20 & 500 & 0.45 & 2200 & 0.020 \\
Stiff WIB & 5000 & 0.20 & 2400 & \\
\hline
\end{tabular}

Table 1: Properties of the soil and the stiff WIB.

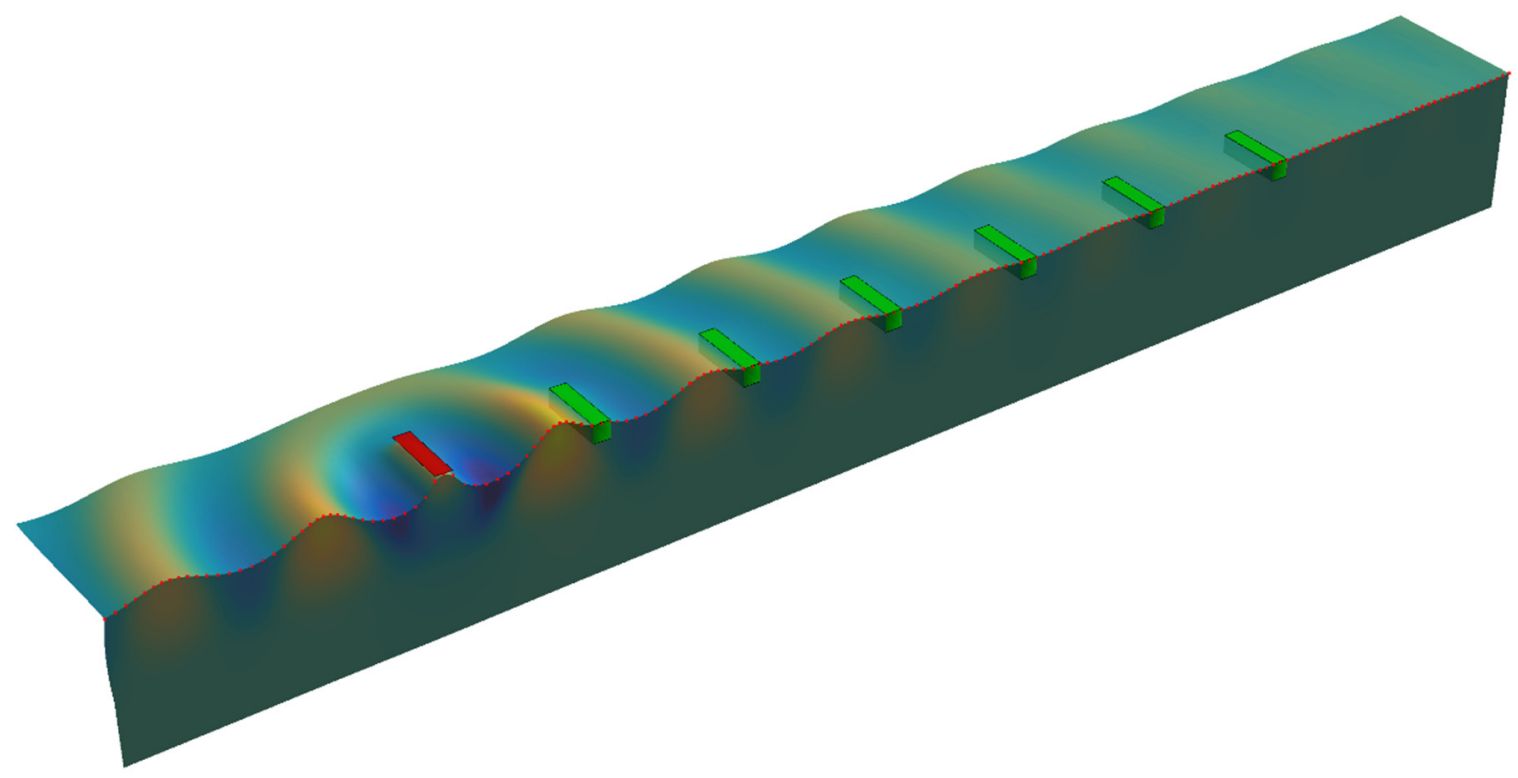

Figure 3: Example results for layered half-space subjected to a harmonic load applied at $6 \mathrm{~Hz}$ on a rigid (red) plate and with six rigid (green) $2 \mathrm{~m}$ high blocks embedded $2 \mathrm{~m}$ into the soil. The ground surface and a vertical cut through the centre line of the array are illustrated. Dark blue and bright yellow shades indicate negative and positive vertical displacements, respectively. 


\section{TWO-DIMENSIONAL ANALYSIS OF INFINITE PERIODIC WIB ARRAY}

Often, dispersion diagrams are plotted to obtain a visual representation of the wave modes. A dispersion diagram may provide an overview of the number of waves that propagate in the structure or medium, but information about attenuated or evanescent modes is lost. As proposed by Andersen [1], a count of different modes occurring at a various frequencies can be presented by a "skyline plot". Using this type of diagram, Figure 4 shows the results obtained for periodic 2D models of a WIB array with different configurations. Only modes with imaginary parts of the wavenumber smaller than $1.0 \mathrm{~m}^{-1}$, i.e. small degree of attenuation, have been included.
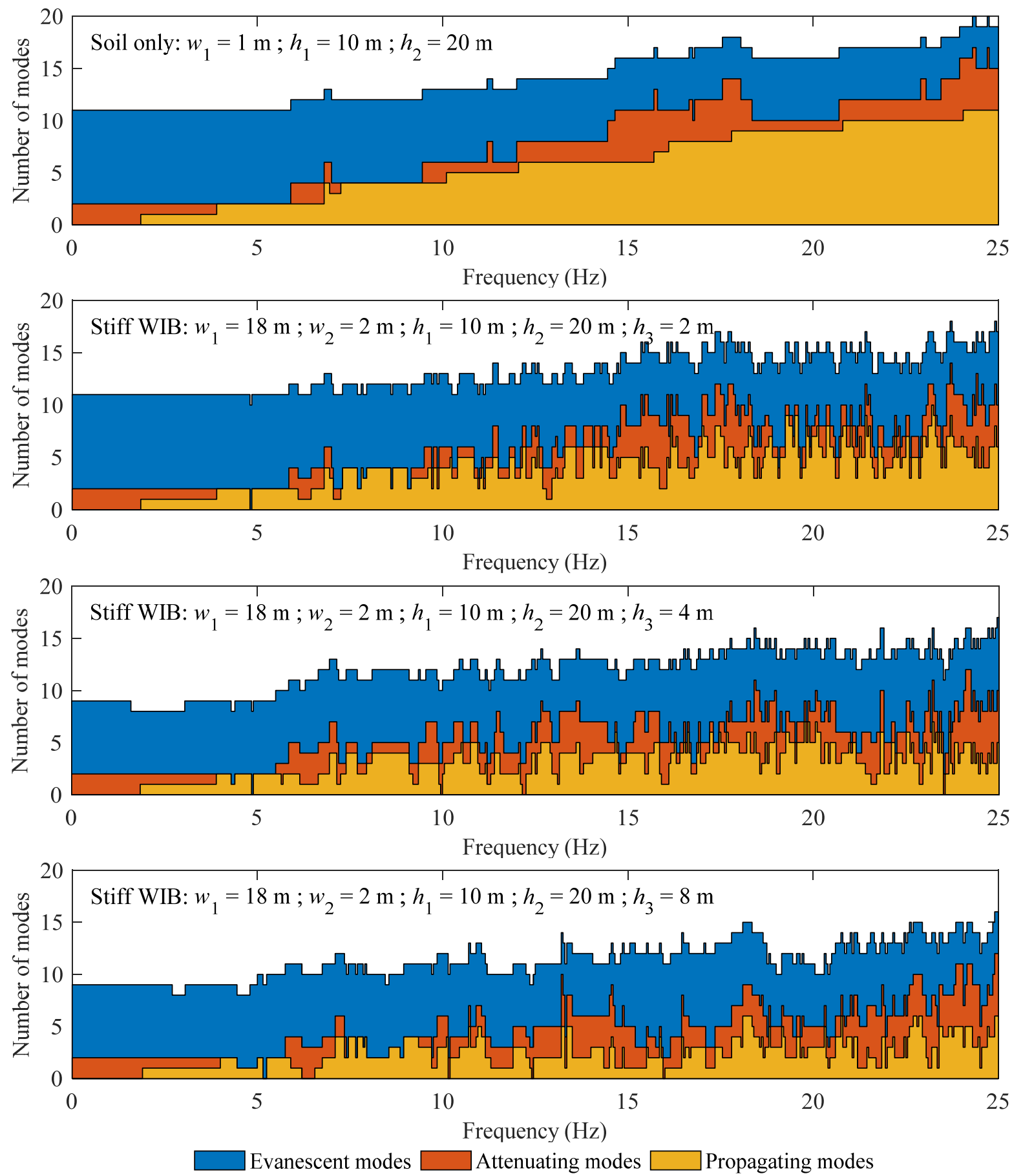

Figure 4: Number of evanescent, attenuating and propagating modes in two-dimensional models of two-layered soil with different depths of a $2 \mathrm{~m}$ wide WIB placed periodically at a centre-to-centre distance of $18 \mathrm{~m}$. 
Although only modes with low attenuation have been included, it is clear from Figure 4 that the sum of attenuating and evanescent modes dominates the transmission of vibration energy in the first case with soil only-especially at low frequencies. Above the cut-on frequency, about $2 \mathrm{~Hz}$, the number of propagating modes constitutes about one-quarter of all transmission modes. An increase from a single propagating mode at $2 \mathrm{~Hz}$ to 10 propagating modes at $20 \mathrm{~Hz}$ is observed with a similar linearly increasing trend for the lowly damped attenuating and evanescent modes. Importantly, above the cut-on frequency propagating modes (and hence propagating energy) exist for all frequencies in the reference case where no WIBs are present.

The three lower subfigures of Figure 4 show the effect on the modal propagation due to the inclusion of an increasing sized obstacle. The obstacle lies just under the surface and increases in depth from $2 \mathrm{~m}$ to $8 \mathrm{~m}$. At $8 \mathrm{~m}$ depth, the obstacle is $2 \mathrm{~m}$ from the interface between the two layers. It is immediately clear that a stopband appears close to $5 \mathrm{~Hz}$, which then shifts slightly and increases in width as the object inclusions increase in size. An additional stopband occurs around $6 \mathrm{~Hz}$, and it can be observed that few attenuated modes are present near $8 \mathrm{~Hz}$. This provides the general picture that mitigation of vibration in the frequency range from about 4 to $8 \mathrm{~Hz}$ may be achieved by the periodic WIB array. Further stopbands appear at $10 \mathrm{~Hz}, 12 \mathrm{~Hz}$ and $16 \mathrm{~Hz}$ for the $8 \mathrm{~m}$ deep inclusion. Overall, the stopbands evolve gradually. Thus, with increasing embedment depth and height of the blocks, fewer propagating modes and lightly damped attenuated modes exist - also outside the stopbands. This suggests that even an array that does not lead to true stopbands (in the sense that no waves at all propagate) may still be effective regarding vibration mitigation.

\section{THREE-DIMENSIONAL ANALYSIS OF FINITE WIB ARRAYS}

This study concerns finite arrays of WIBs that are either placed on the ground surface or embedded within the stratum. The soil is layered as outlined in Table 1 and as mentioned in Section 2.4, the WIBs are rigid blocks with a width of $18 \mathrm{~m}$ in the transverse direction and a length of $2 \mathrm{~m}$ in the longitudinal direction, i.e. along the array. Unless defined otherwise, the WIBs are placed equidistantly with a centre-to-centre distance of $18 \mathrm{~m}$ as within the 2D model analysed in Section 3. The objective is to demonstrate the effectiveness of WIBs in 3D along the ground surface at certain frequencies, and as function of frequency at certain receiver points.

\subsection{Results for WIB arrays with a block height of $\mathbf{2} \mathbf{m}$ and different embedment}

Arrays of 2, 4 or 6 WIBs with a block height of $2 \mathrm{~m}$ are considered. The blocks are either resting on the ground surface or embedded $2 \mathrm{~m}$ or $4 \mathrm{~m}$ into the soil. In the latter configuration, the WIBs are thus fully buried with $2 \mathrm{~m}$ of soil over the blocks. For each embedment depth, the insertion loss (IL) is calculated at frequencies up to $25 \mathrm{~Hz}$ and for distances up to $125 \mathrm{~m}$ from the load, which covers practical ranges receiver distances for vibrations from rail traffic.

For each case, results are presented for the three frequencies $5 \mathrm{~Hz}, 10 \mathrm{~Hz}$ and $20 \mathrm{~Hz}$ for all positions along the centre line of the array on the ground surface up to $125 \mathrm{~m}$ from the load. Further, results are presented at frequencies up to $25 \mathrm{~Hz}$ at three receiver positions: $45 \mathrm{~m}, 81 \mathrm{~m}$ and $117 \mathrm{~m}$. When first studied, large oscillations in the response were observed as result of small changes in frequency or position. Plotting the raw results at a single frequency or position may therefore provide misleading judgement of the overall mitigation effects. Hence, in the results shown for single receiver positions, the vertical magnitude of displacements has been averaged over 9 points along the centre line of the array. The distance between the points is $1 \mathrm{~m}$ and the receiver point (e.g. $x=45 \mathrm{~m}$ ) is placed in the middle. This way, the ground surface is analysed over an area that may represent the footprint of a typical building. Likewise, result presented for single frequencies have been averaged over a range of frequencies $\pm 1 \mathrm{~Hz}$. 
Figure 5 and Figure 6 show the IL achieved with different arrays of $2 \mathrm{~m}$ high blocks placed on the ground surface, whereas Figure 7 and Figure 8 show similar results for $2 \mathrm{~m}$ high blocks embedded fully, i.e. to a depth of $2 \mathrm{~m}$. Finally, Figure 9 and Figure 10 show the IL obtained with arrays of $2 \mathrm{~m}$ high blocks embedded $4 \mathrm{~m}$ into the ground. As a general trend, the WIB arrays provide positive IL, and there is a clear effect of changing the embedment depth or adding more WIBs to the array. The introduction of a rigid block effectively modifies the layer depth and stiffness properties over a finite portion of the ground, although blocks up to $107 \mathrm{~m}$ from the load are studied here, so it could be said to represent an approximate global variation in space. Hence, the modification of the layered ground below the surface would raise the cuton frequency and thereby modify the vibration response at certain frequencies.

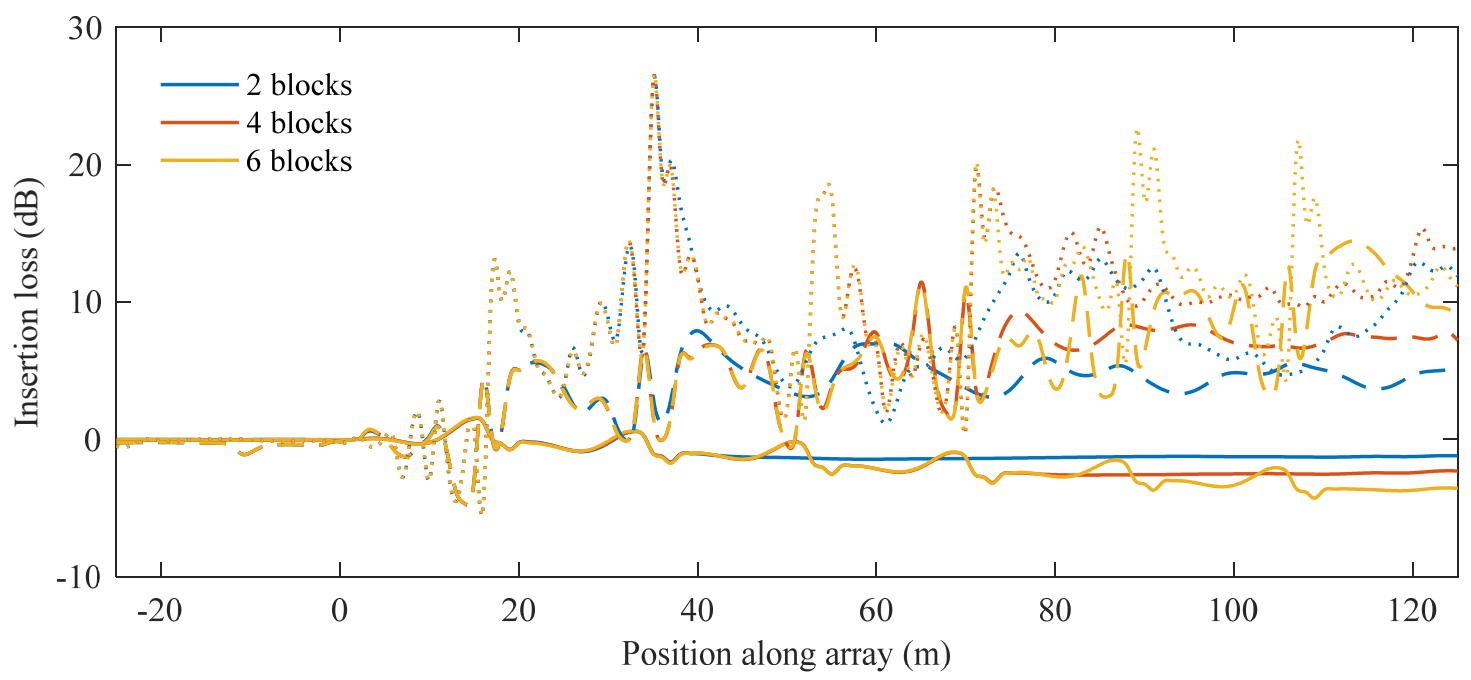

Figure 5. Insertion loss as function of position for $2 \mathrm{~m}$ high WIBs embedded $0 \mathrm{~m}$ into a two-layer stratum. Full lines (-) indicate results at $5 \mathrm{~Hz}$; dashed lines $(--)$ indicate results at $10 \mathrm{~Hz}$; dotted lines $(\cdots \cdots)$ indicate results at $20 \mathrm{~Hz}$.

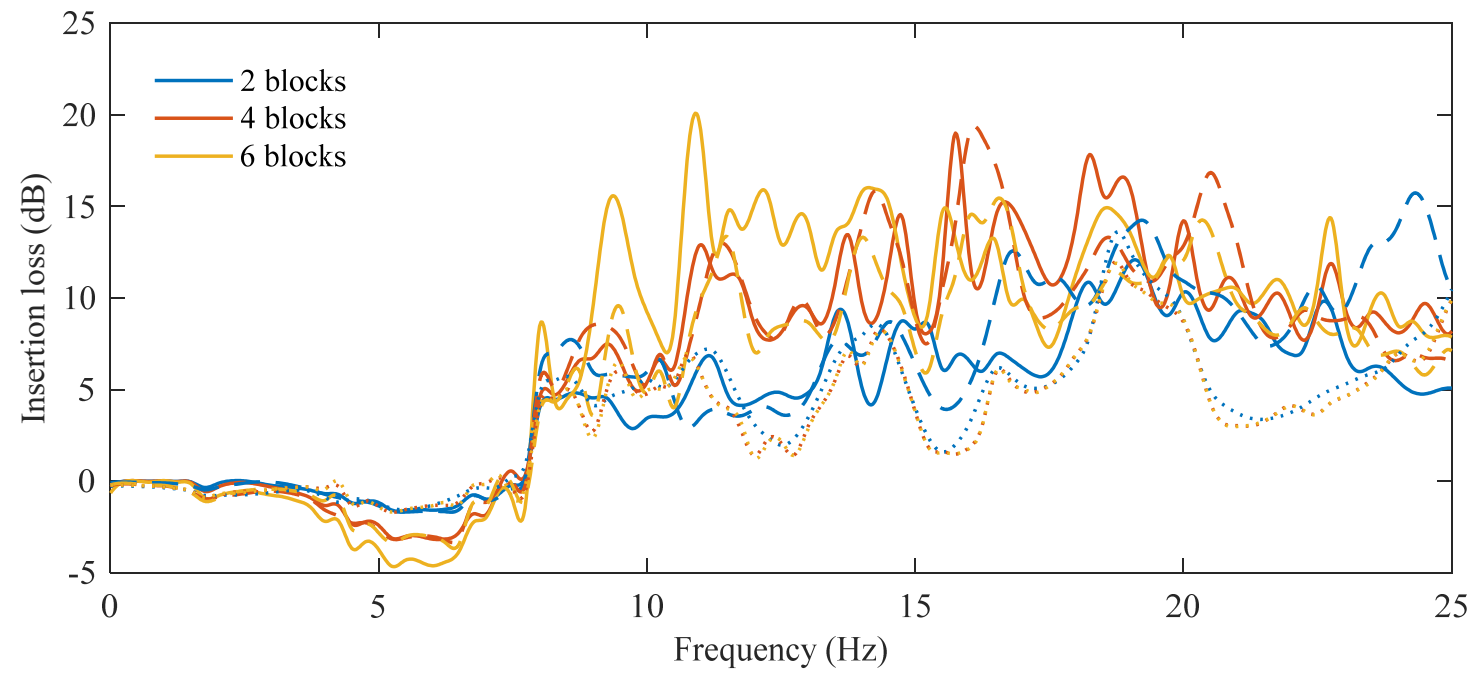

Figure 6: Insertion loss as function of frequency for $2 \mathrm{~m}$ high WIBs embedded $0 \mathrm{~m}$ into a two-layer stratum.

Full lines (-) indicate results at $x=117 \mathrm{~m}$; dashed lines $(--)$ indicate results at $x=81 \mathrm{~m}$; dotted lines $(\cdots \cdots)$ indicate results at $x=45 \mathrm{~m}$.

According to Figure 5 and Figure 6, the blocks placed on the ground surface provide an IL of about $10-15 \mathrm{~dB}$ at frequencies higher than $10 \mathrm{~Hz}$. Especially, there are signs of stopband 
behaviour as the number of blocks is increased, from 2 to 4 to 6 blocks, near $9 \mathrm{~Hz}$ and again near $12 \mathrm{~Hz}$. The waviness of the IL is due to the patterns of destructive interference beyond the block locations. Below $4 \mathrm{~Hz}$, close to cut-on, the effect of the blocks is marginal. However, at intermediate frequencies between $4 \mathrm{~Hz}$ and $8 \mathrm{~Hz}$ (where the wide stopband was detected in the periodic 2D array of embedded WIBs) the blocks on the ground surface have a poor effect, leading to increasingly negative IL with increasing numbers of blocks. Obviously, this effect must be avoided, given the intention of mitigating rather than amplifying the vibration.

In Figure 7 and Figure 8, the blocks are submerged into the upper soil layer by $2 \mathrm{~m}$. This changes the IL completely at low frequencies compared the previous case with blocks placed on the ground surface. Thus, with the blocks embedded just below the surface, the effect on the reduction of vibration is clear. The modification of modal wave propagation is clearly seen, and an IL of more than $10 \mathrm{~dB}$ occurs in the frequency range from $4 \mathrm{~Hz}$ to $8 \mathrm{~Hz}$ when all six blocks are present. Further, as expected, the addition of more blocks leads to increasing IL.

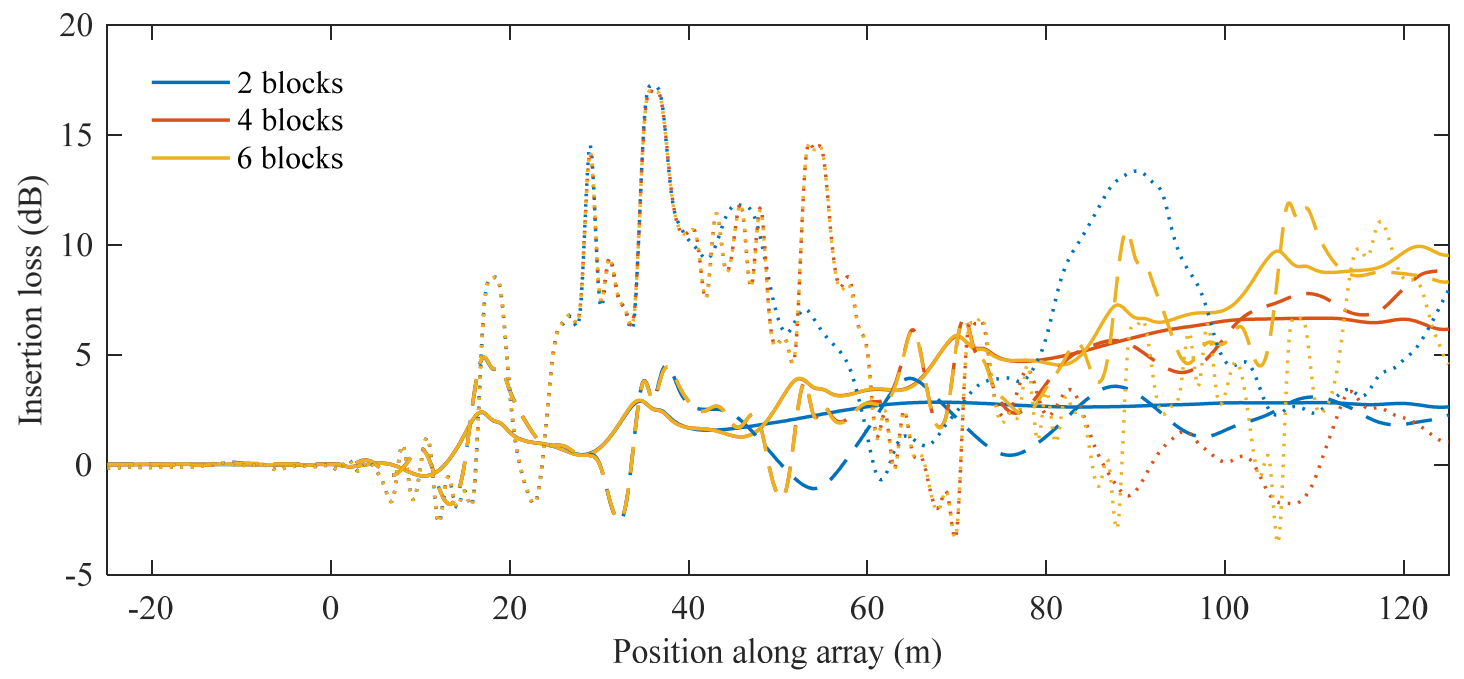

Figure 7: Insertion loss as function of position for $2 \mathrm{~m}$ high WIBs embedded $2 \mathrm{~m}$ into a two-layer stratum. Full lines (-) indicate results at $5 \mathrm{~Hz}$; dashed lines $(--)$ indicate results at $10 \mathrm{~Hz}$; dotted lines $(\cdots \cdots)$ indicate results at $20 \mathrm{~Hz}$.

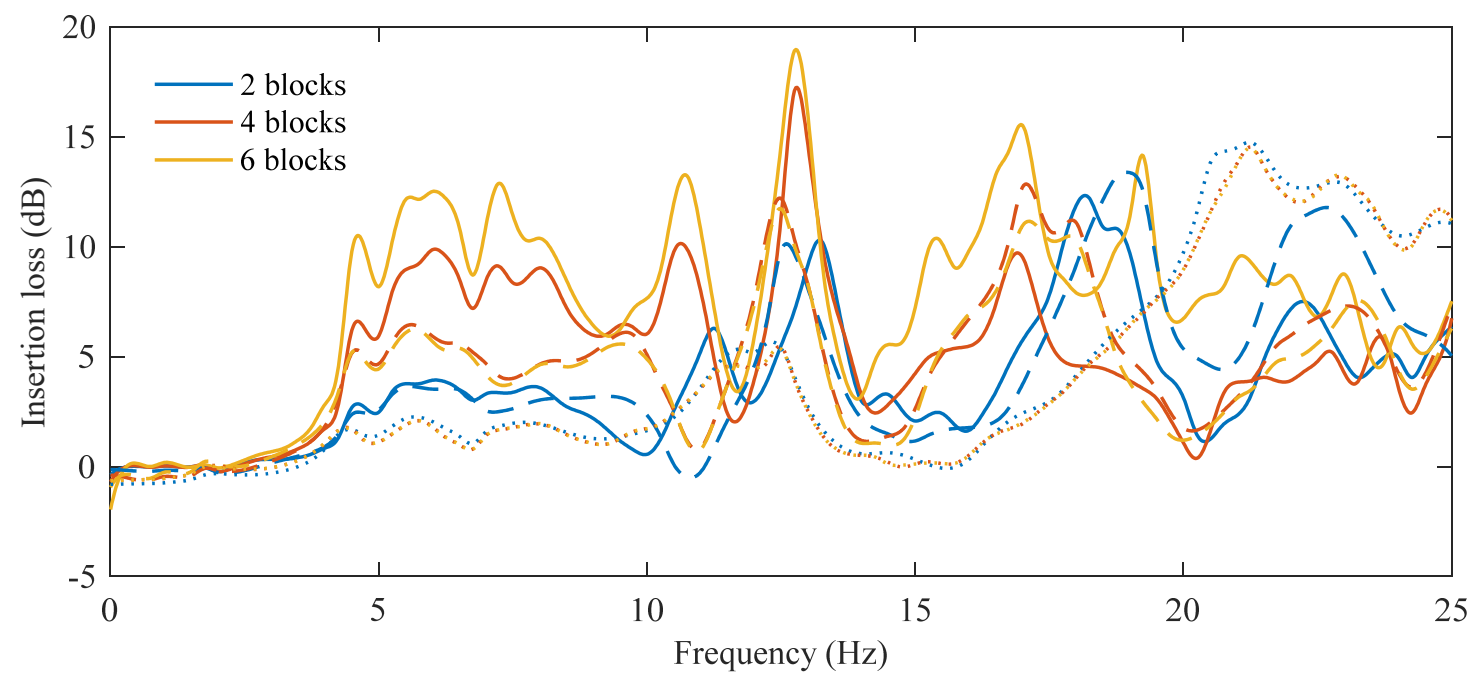

Figure 8: Insertion loss as function of frequency for $2 \mathrm{~m}$ high WIBs embedded $2 \mathrm{~m}$ into a two-layer stratum.

Full lines (-) indicate results at $x=117 \mathrm{~m}$; dashed lines $(--)$ indicate results at $x=81 \mathrm{~m}$; dotted lines $(\cdots \cdots)$ indicate results at $x=45 \mathrm{~m}$. 
Referring to Figure 9 and Figure 10, the WIB arrays with the $2 \mathrm{~m}$ high blocks embedded $4 \mathrm{~m}$ into the soil have a performance equal to the WIB arrays with 2 m embedment depth. However, interesting differences occur. Compared to the IL reported in Figure 5, Figure 9 shows a much lower IL at the frequency $20 \mathrm{~Hz}$ for positions around 20-60 m. Here, there is a large degree of similarity between the cases with blocks on the ground surface (Figure 5) or embedded two metres (Figure 7), but a completely different behaviour occurs with the WIBs embedment four metres into the ground (Figure 9). However, at positions beyond $60 \mathrm{~m}$, the WIB array with $4 \mathrm{~m}$ embedment provides the better alternative with positive IL at all positions and frequencies. By contrast, the blocks on the surface lead to negative IL for all positions in the range at low frequencies (see results at $5 \mathrm{~Hz}$ in Figure 5), and negative IL is observed at $20 \mathrm{~Hz}$ right in front of the WIBs embedded $2 \mathrm{~m}$ into the ground (see Figure 7).

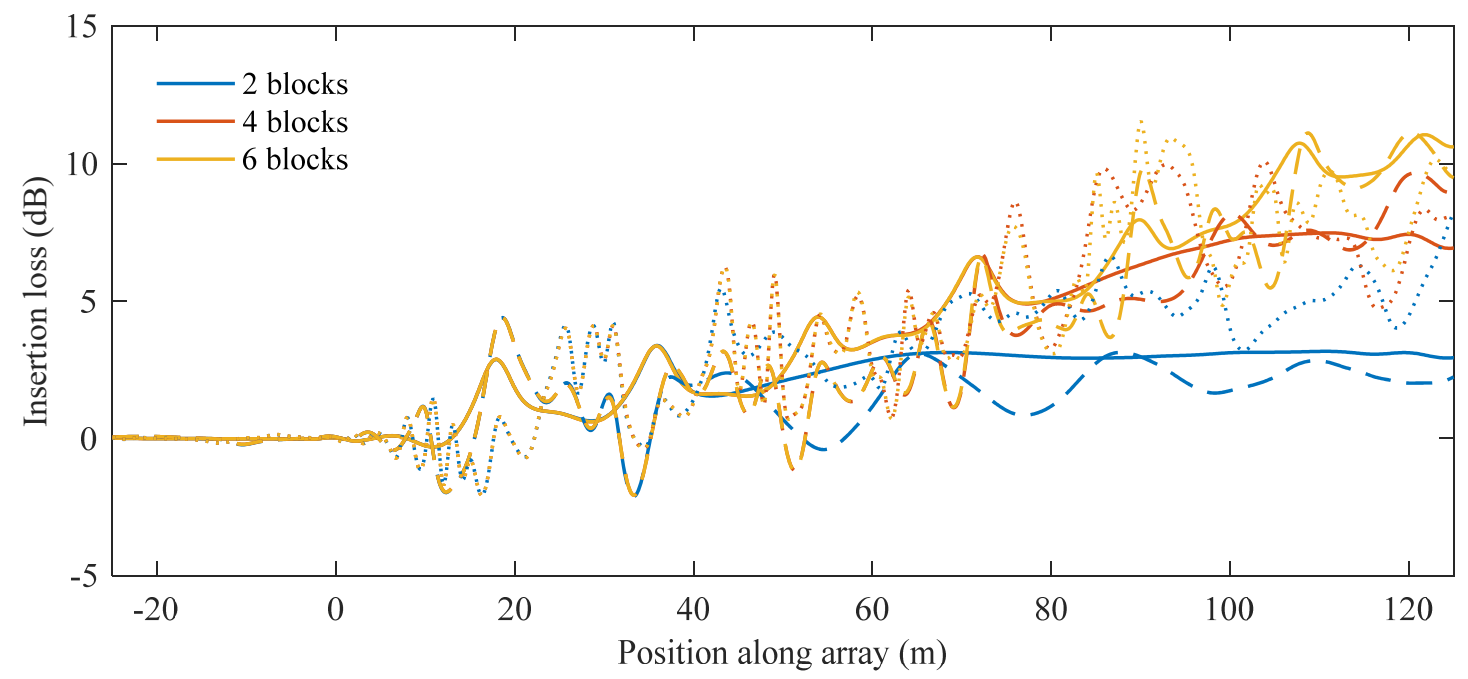

Figure 9: Insertion loss as function of position for $2 \mathrm{~m}$ high WIBs embedded $4 \mathrm{~m}$ into a two-layer stratum. Full lines (-) indicate results at $5 \mathrm{~Hz}$; dashed lines $(--)$ indicate results at $10 \mathrm{~Hz}$; dotted lines $(\cdots \cdots)$ indicate results at $20 \mathrm{~Hz}$.

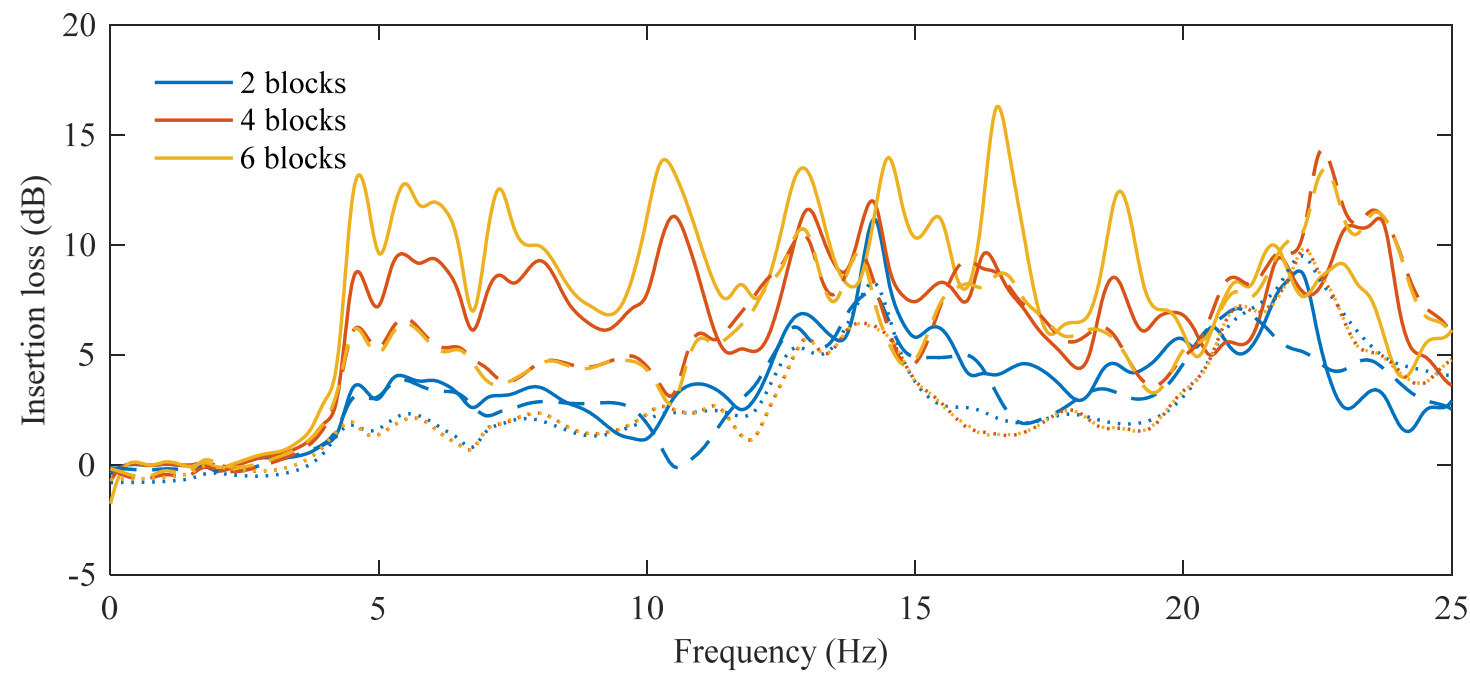

Figure 10: Insertion loss as function of frequency for $2 \mathrm{~m}$ high WIBs embedded $4 \mathrm{~m}$ into a two-layer stratum. Full lines (-) indicate results at $x=117 \mathrm{~m}$; dashed lines $(--)$ indicate results at $x=81 \mathrm{~m}$; dotted lines $(\cdots \cdots)$ indicate results at $x=45 \mathrm{~m}$. 


\subsection{Insertion loss for WIB arrays with block height $\mathbf{4} \mathbf{m}$ and different embedment}

In this section, arrays of 2, 4 or 6 WIBs with a block height of $4 \mathrm{~m}$ are considered. The blocks are again resting on the ground surface, partially embedded to $2 \mathrm{~m}$ depth, or fully embedded to a depth of $4 \mathrm{~m}$. The IL obtained for the different configurations are presented in Figures 11-16. Firstly, compared to the previous case with $2 \mathrm{~m}$ high blocks, a greater IL is observed in most cases. For the cases with four or six $4 \mathrm{~m}$ high blocks fully embedded in the ground, Figure 16 shows an IL of more than $15 \mathrm{~dB}$ at $x=117 \mathrm{~m}$ in the frequency range $4-8 \mathrm{~Hz}$, and Figure 15 shows a similar IL at $20 \mathrm{~Hz}$ for some positions in the range $20-50 \mathrm{~m}$.

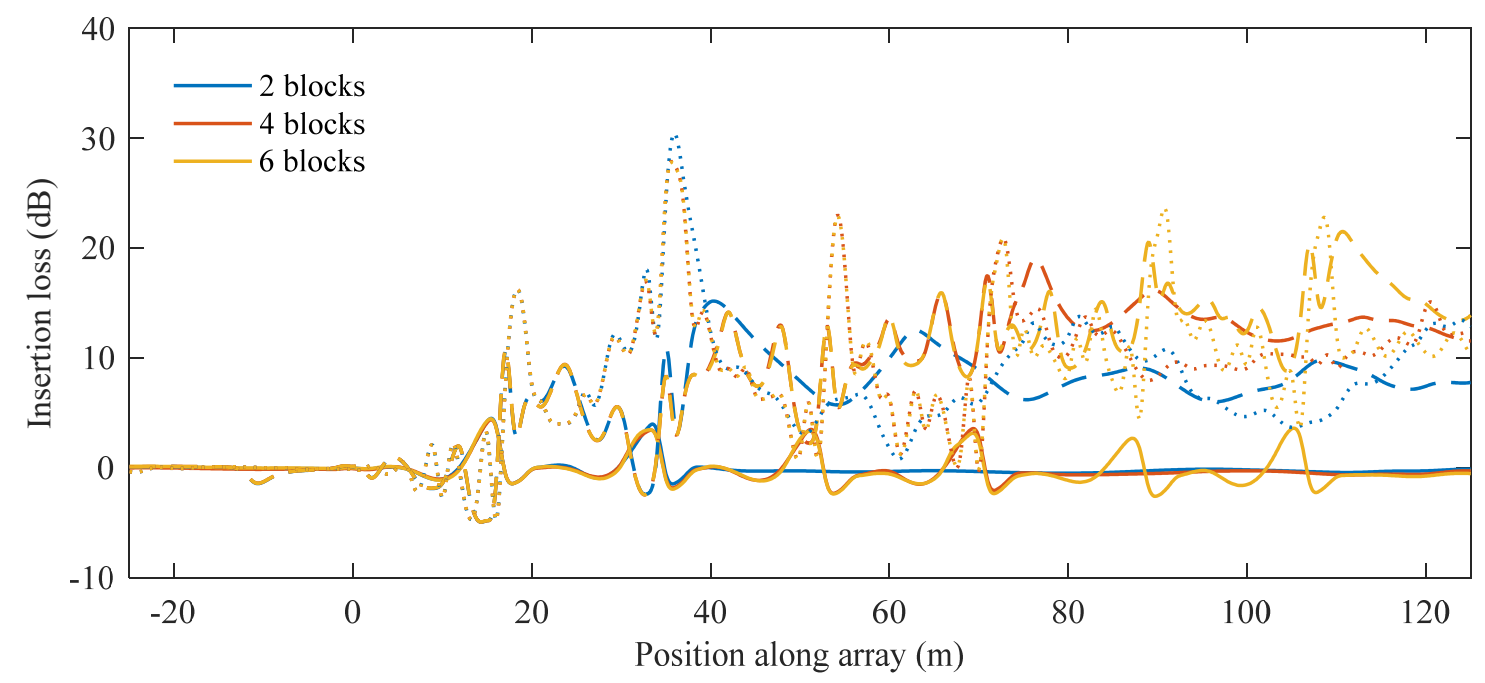

Figure 11: Insertion loss as function of position for $4 \mathrm{~m}$ high WIBs embedded $0 \mathrm{~m}$ into a two-layer stratum. Full lines $(-)$ indicate results at $5 \mathrm{~Hz}$; dashed lines $(--)$ indicate results at $10 \mathrm{~Hz}$; dotted lines $(\cdots \cdots)$ indicate results at $20 \mathrm{~Hz}$.

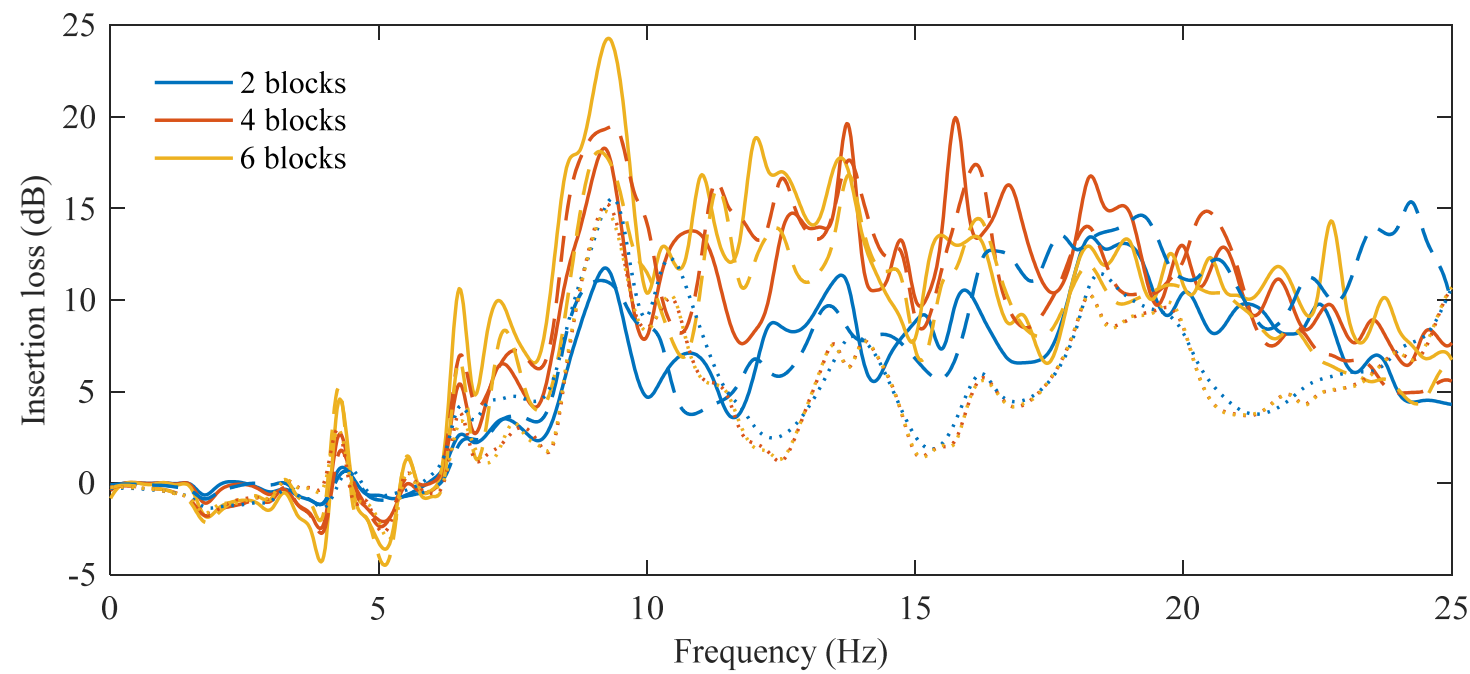

Figure 12: Insertion loss as function of frequency for $4 \mathrm{~m}$ high WIBs embedded $0 \mathrm{~m}$ into a two-layer stratum. Full lines (-) indicate results at $x=117 \mathrm{~m}$; dashed lines $(--)$ indicate results at $x=81 \mathrm{~m}$; dotted lines $(\cdots \cdots)$ indicate results at $x=45 \mathrm{~m}$.

Comparing Figure 6 to Figure 12, it can be observed that the taller blocks have much stronger influence on the vibration at frequencies below $4 \mathrm{~Hz}$ than the lower blocks. Especially, variations of about $\pm 4 \mathrm{~dB}$ occur around $4 \mathrm{~Hz}$ for the block height of $4 \mathrm{~m}$, indicating presence 
of a resonance frequency related to block motion. For the $2 \mathrm{~m}$ high WIBs, negative IL occurred at $x=117 \mathrm{~m}$ in the entire frequency range from $4 \mathrm{~Hz}$ to $8 \mathrm{~Hz}$. This is not the case with the WIB height $4 \mathrm{~m}$. In continuation of this, the taller WIBs provide less negative IL for all positions in the range 60-125 m compared with the lower WIBs (see Figure 5 and Figure 11).

As a deviation from the general trend, a comparison of Figure 7 and Figure 8 with Figure 13 and Figure 14 reveals that the $4 \mathrm{~m}$ high blocks embedded $2 \mathrm{~m}$ into the soil provide a smaller IL than the $2 \mathrm{~m}$ high blocks embedded to the same depth. This is in particular the case at positions around $x=45 \mathrm{~m}$ and at frequencies above $20 \mathrm{~Hz}$. Figure 14 clearly shows stopband behaviour at $x=117 \mathrm{~m}$ for the frequencies $6 \mathrm{~Hz}, 8 \mathrm{~Hz}$ and $10 \mathrm{~Hz}$. These frequencies were also identified as stopbands in the $2 \mathrm{D}$ model of the infinite periodic array, or as frequencies at which few propagating and attenuating modes exist when WIBs of large size are present.

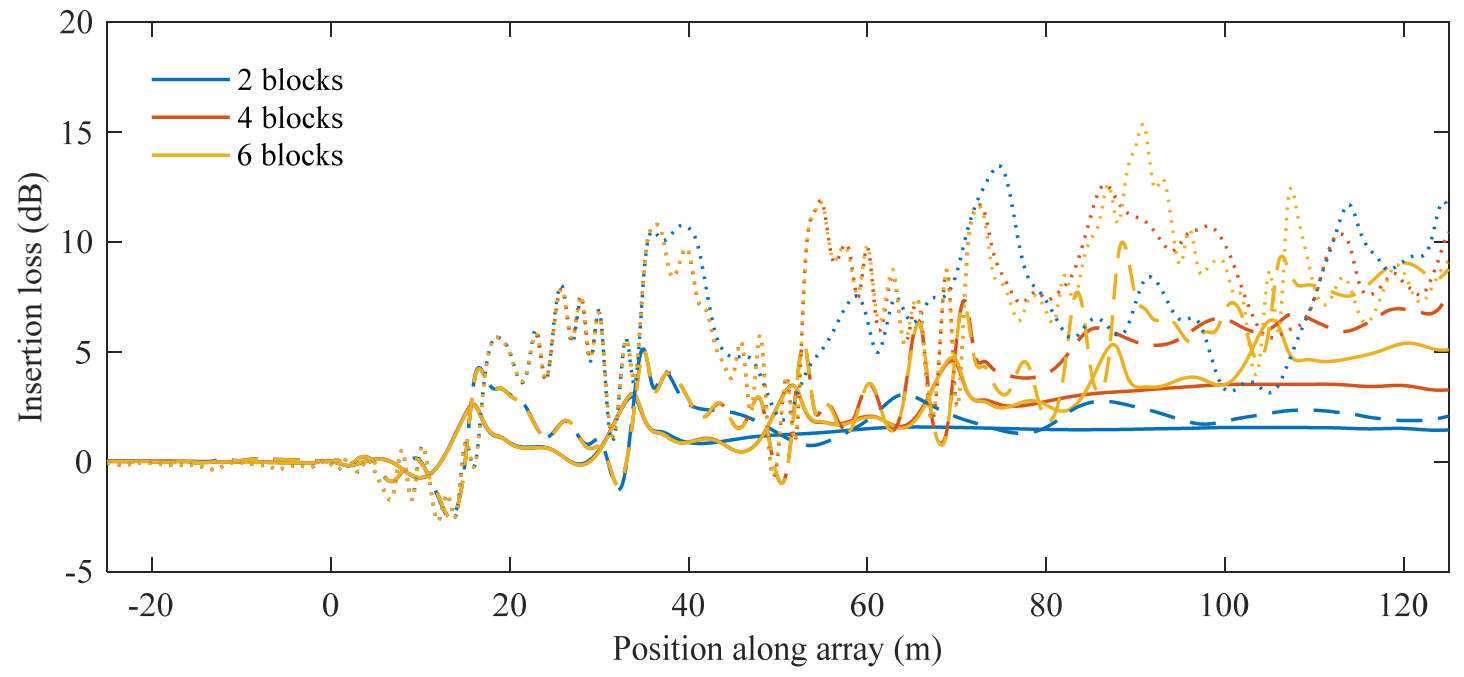

Figure 13: Insertion loss as function of position for $4 \mathrm{~m}$ high WIBs embedded $2 \mathrm{~m}$ into a two-layer stratum. Full lines (-) indicate results at $5 \mathrm{~Hz}$; dashed lines $(--)$ indicate results at $10 \mathrm{~Hz}$; dotted lines $(\cdots \cdots)$ indicate results at $20 \mathrm{~Hz}$.

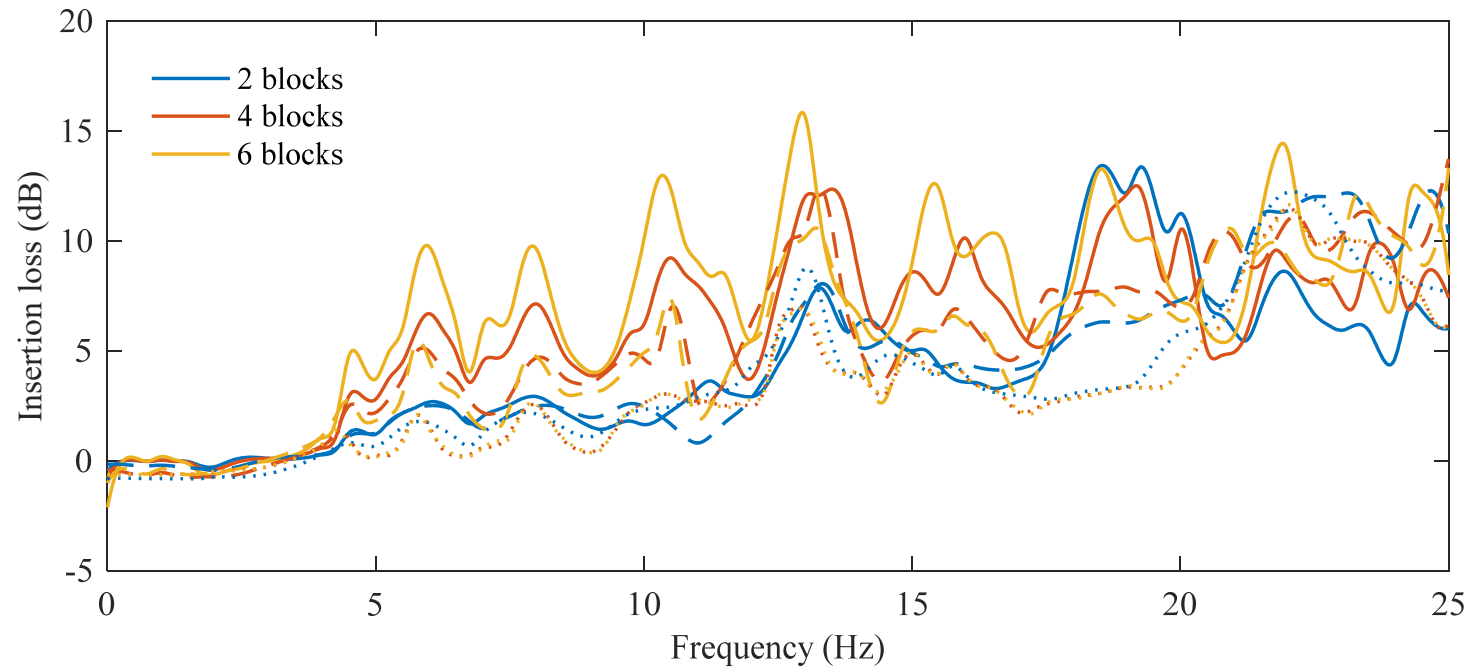

Figure 14: Insertion loss as function of frequency for $4 \mathrm{~m}$ high WIBs embedded $2 \mathrm{~m}$ into a two-layer stratum. Full lines (-) indicate results at $x=117 \mathrm{~m}$; dashed lines $(--)$ indicate results at $x=81 \mathrm{~m}$; dotted lines $(\cdots \cdots)$ indicate results at $x=45 \mathrm{~m}$. 


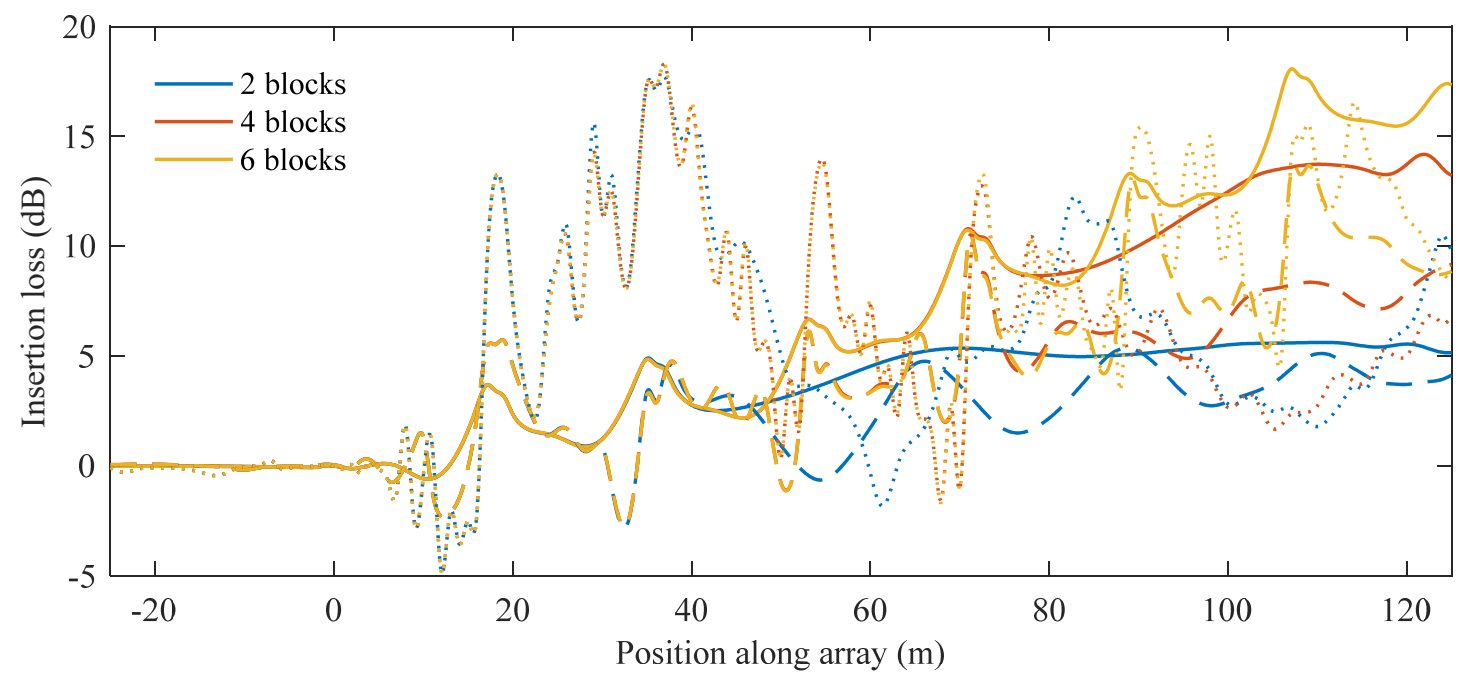

Figure 15: Insertion loss as function of position for $4 \mathrm{~m}$ high WIBs embedded $4 \mathrm{~m}$ into a two-layer stratum. Full lines (-) indicate results at $5 \mathrm{~Hz}$; dashed lines $(--)$ indicate results at $10 \mathrm{~Hz}$; dotted lines $(\cdots \cdots)$ indicate results at $20 \mathrm{~Hz}$.

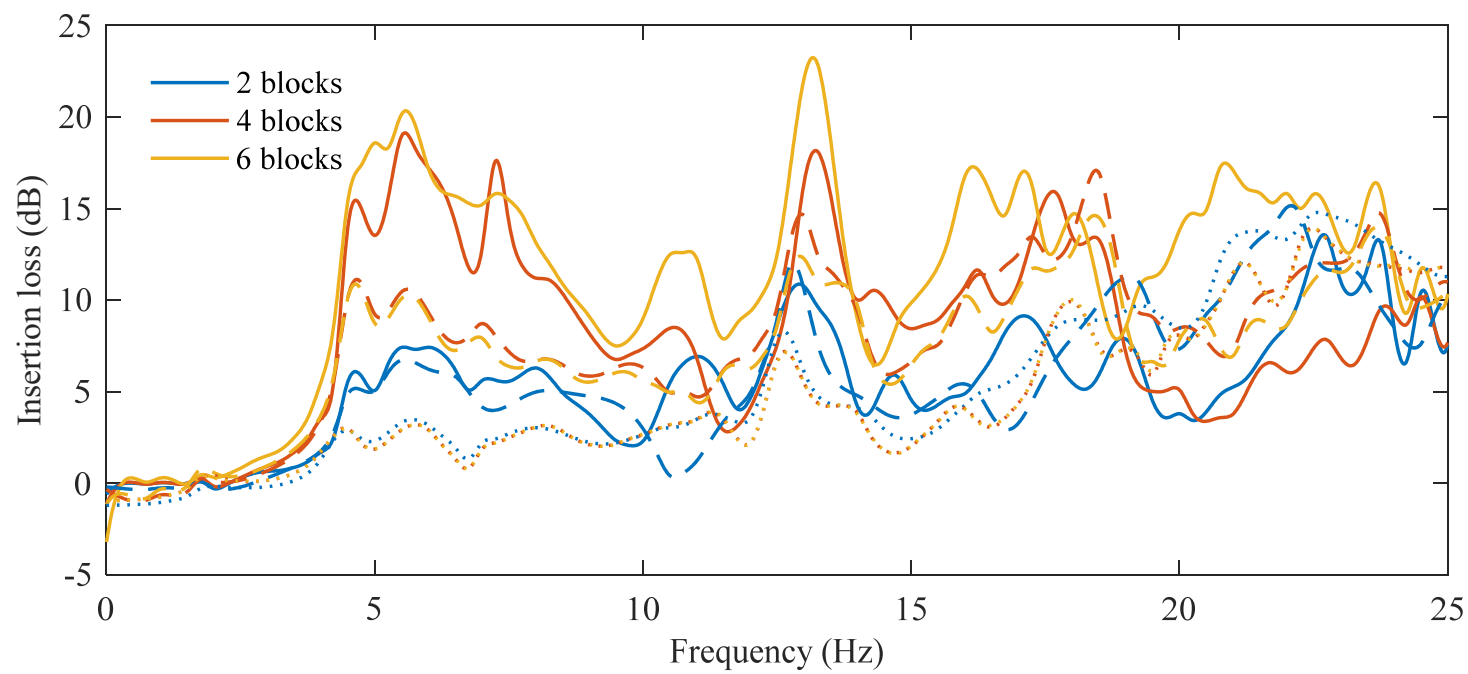

Figure 16: Insertion loss as function of frequency for $4 \mathrm{~m}$ high WIBs embedded $4 \mathrm{~m}$ into a two-layer stratum.

Full lines (-) indicate results at $x=117 \mathrm{~m}$; dashed lines $(--)$ indicate results at $x=81 \mathrm{~m}$; dotted lines $(\cdots \cdots)$ indicate results at $x=45 \mathrm{~m}$.

Finally, the WIB arrays with $4 \mathrm{~m}$ high blocks fully embedded in the ground provide higher IL than any other configuration tested in the present analyses. The IL of $15 \mathrm{~dB}$ reported by Figure 15 and Figure 16 is significant and demonstrates that this type of solution can be very effective in mitigating vibration from, for example, railways. Remarkably, the effect is high in the frequency range from $4-8 \mathrm{~Hz}$, where most other mitigation concepts, e.g. single trenches or wave barriers, fail to provide much reduction in vibration level.

When comparing the configurations with block height $4 \mathrm{~m}$ to those with block height $2 \mathrm{~m}$, one idea is to look at cases with the same embedment depth. However, it may be more meaningful to compare cases, in which the WIBs are fully embedded to a depth matching their height. In accordance with this idea, it can be observed that the WIB arrays with embedment depth and block height both equal to $4 \mathrm{~m}$ (Figure 15 and Figure 16) provide insertion losses that are in most aspects similar to, but higher than those achieved with embedment depth and block height both equal to $2 \mathrm{~m}$ (Figure 7 and Figure 8). With these configurations, no part of the WIBs stick 
out of the ground, but since the blocks go all the way to the ground surface, short surface waves (at high frequencies) cannot pass above the WIBs. This comes in addition to the full influence of the rigid blocks towards increasing the stiffness of the ground. Thus, when the blocks are exactly fully embedded, they have two features: 1) they serve as wave barriers at high frequencies; 2) they add stiffness preventing wave propagation at low frequencies.

\subsection{Influence of variations in the distances between WIBs}

A periodic structure, in this case the array of WIBs, may produce stopbands as an effect of negative interference of waves. In order to analyse to what extent the mitigation efficiency of the array diminishes when the WIBs are not placed equidistantly, six configurations with small perturbations of the WIB positions are compared with a reference case in which four WIBs are placed repeatedly with a centre-to-centre distance of $18 \mathrm{~m}$. The positions of the four WIBs in the reference case and each of the six variations are given in Table 2.

\begin{tabular}{lllll}
\hline & WIB $1[\mathrm{~m}]$ & WIB 2 [m] & WIB 3 [m] & WIB 4 [m] \\
\hline Reference case & 18.0 & 36.0 & 54.0 & 72.0 \\
Variation 1 & 16.5 & 34.0 & 52.5 & 72.0 \\
Variation 2 & 16.5 & 36.0 & 53.5 & 72.0 \\
Variation 3 & 17.5 & 35.0 & 53.5 & 72.0 \\
Variation 4 & 17.5 & 36.0 & 53.5 & 72.0 \\
Variation 5 & 17.0 & 34.0 & 53.0 & 72.0 \\
Variation 6 & 16.5 & 36.0 & 53.0 & 72.0 \\
\hline
\end{tabular}

Table 2: Positions of WIBs in reference case and in variations 1-6.

Figure 17 shows the IL as function of position along the WIB arrays defined by Table 2. In all cases, the WIBs are $2 \mathrm{~m}$ high and embedded $2 \mathrm{~m}$ into the ground and the figure shows results obtained at the frequencies 5,10 and $20 \mathrm{~Hz}$. Clearly, at the lower frequencies little difference occurs due to the changes in positions of individual WIBs. Beyond the last WIB, i.e. at positions greater than $72 \mathrm{~m}$, the insertion losses at $5 \mathrm{~Hz}$ are close to being identical for the reference case and all six variations. At $10 \mathrm{~Hz}$, the differences are below $1 \mathrm{~dB}$ behind the last WIB, but at $20 \mathrm{~Hz}$, differences up to about $5 \mathrm{~dB}$ occur. However, the general impression is that small variations in the positions of the individual blocks, as defined in Table 2, will not lead to significant changes in the overall performance of the WIB array. However, before a general conclusion can be drawn in this regard, the IL at more frequencies must be analysed.

Figure 18 shows insertion losses for the reference case and the six variations of the WIB array as function of the frequency at three positions. Note that the positions $x=117 \mathrm{~m}$ and $x=81 \mathrm{~m}$ are both behind the last WIB which is placed at the position $72 \mathrm{~m}$ in all cases. Clearly, at all three positions the IL is influenced very little at frequencies up to $9 \mathrm{~Hz}$, meaning that the stopband developing in the frequency range from about 5 to $9 \mathrm{~Hz}$ is unaffected by small perturbations in the WIB positions. At frequencies higher than $10 \mathrm{~Hz}$, some differences occur in the IL related to the various configurations of the WIB array. The IL differs as much as $7 \mathrm{~dB}$ in the frequency range from 20 to $25 \mathrm{~Hz}$ - especially at the position $x=45 \mathrm{~m}$. Here, the reference case with equidistant positions of the blocks provide the higher IL, while variations 1 and 2 (where the first block is placed at $16.5 \mathrm{~m}$ instead of $18.0 \mathrm{~m}$ ) provide the smaller IL. However, beyond the WIB array, i.e. at the positions $x=117 \mathrm{~m}$ and $x=81 \mathrm{~m}$, the differences are slightly smaller. Here absolute differences of about 3-5 dB occur, but this time the reference case provides an IL that is lower than most of the variation. Since the IL for these combinations of positions and frequencies are generally less than $10 \mathrm{~dB}$, a difference of 3-5 dB may be considered important. 
This leads to the conclusion that the positions of the individual WIBs in an array should be optimized to provide a good solution in a wider frequency range and that placing the blocks equidistantly is not necessarily an advantage.

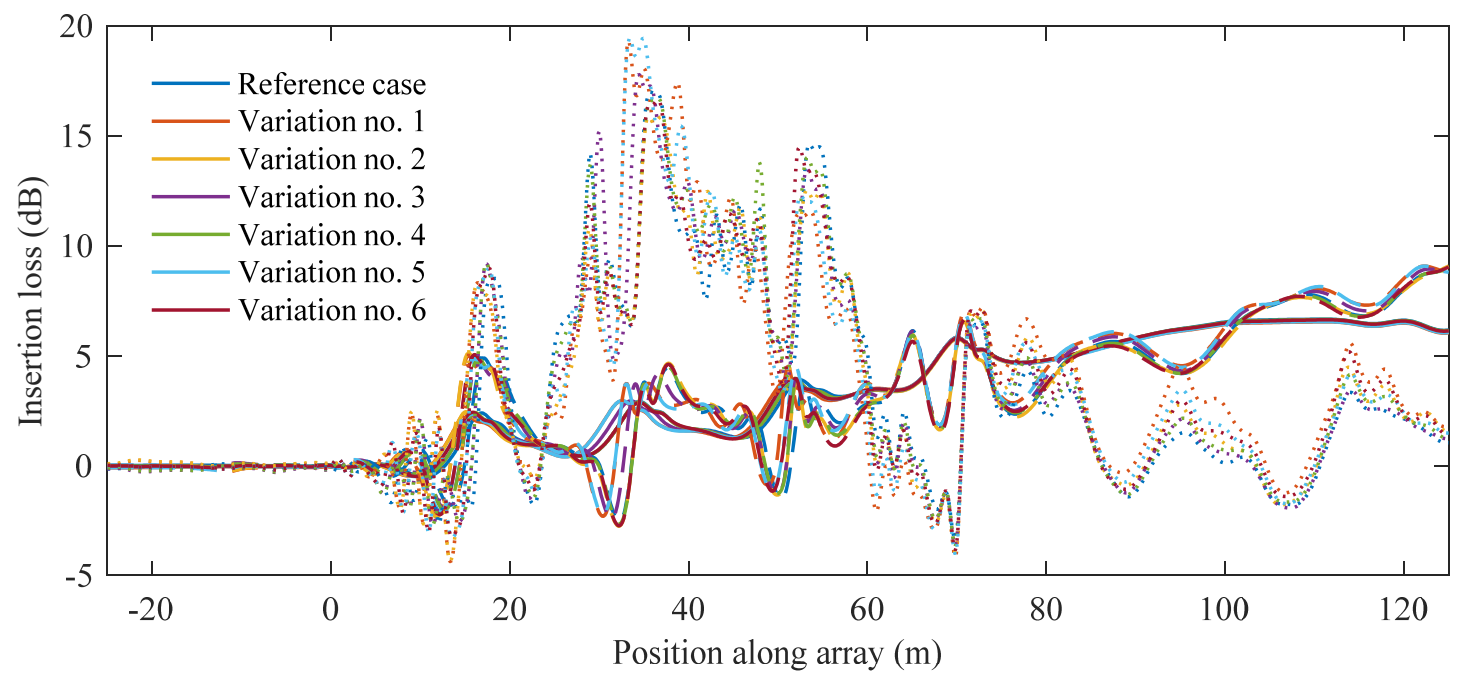

Figure 17: Insertion loss as function of position for $2 \mathrm{~m}$ high WIBs in alternative configurations embedded $2 \mathrm{~m}$ into a two-layer stratum. Full lines (-) indicate results at $5 \mathrm{~Hz}$; dashed lines $(--)$ indicate results at $10 \mathrm{~Hz}$; dotted lines $(\cdots \cdots)$ indicate results at $20 \mathrm{~Hz}$.

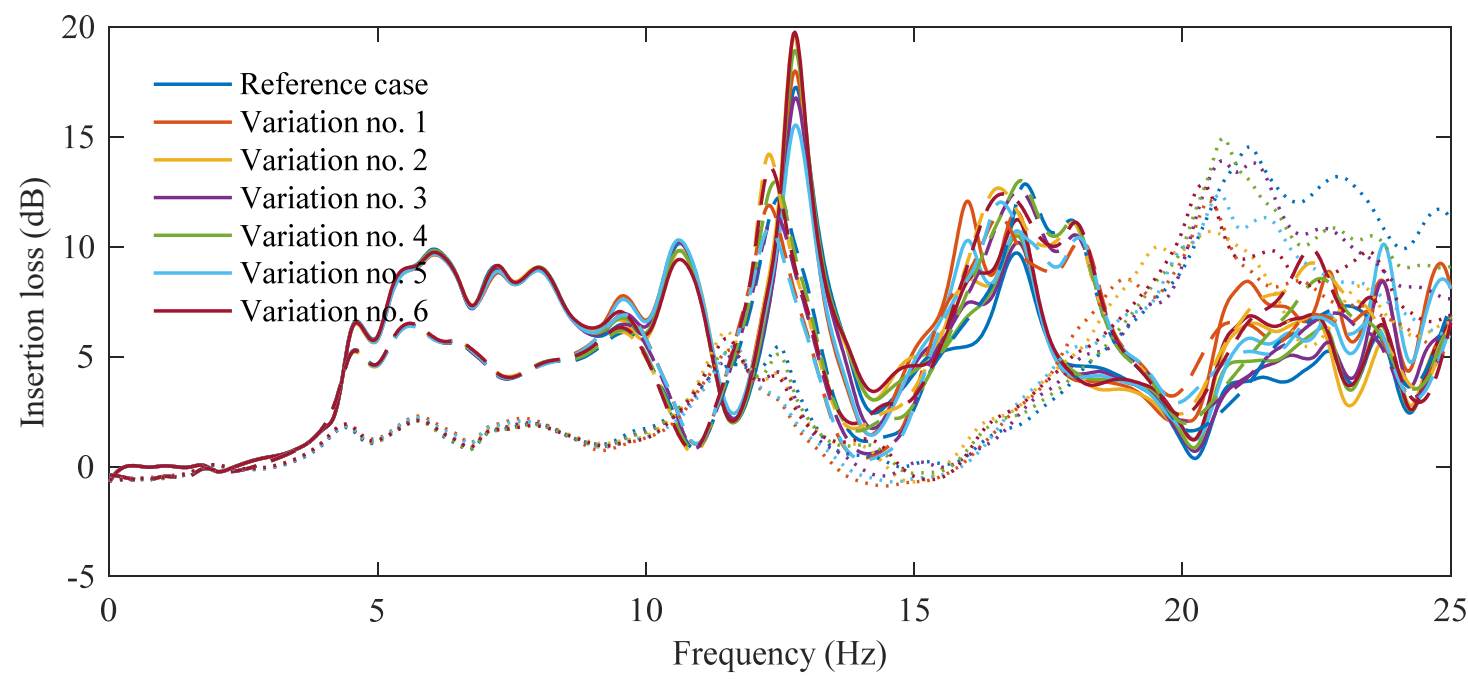

Figure 18: Insertion loss as function of frequency for $2 \mathrm{~m}$ high WIBs in alternative configurations embedded $2 \mathrm{~m}$ into a two-layer stratum. Full lines (-) indicate results at $x=117 \mathrm{~m}$; dashed lines $(--)$ indicate results at $x=81 \mathrm{~m}$; dotted lines $(\cdots \cdots)$ indicate results at $x=45 \mathrm{~m}$.

\subsection{Efficiency of WIB arrays for transient loading}

Intuitively, if an array of WIBs effectively mitigates harmonic waves in the frequency range, mitigation should also occur for transient loads with similar frequency content. To test this, a Ricker wavelet with a magnitude of $1.0 \mathrm{~N}$ and centre frequency $10 \mathrm{~Hz}$ and centre time $0 \mathrm{~s}$ has been applied on the rigid plate. The analysis has been performed by discrete Fourier transformation of the Ricker pulse, based on a sampling rate of $150 \mathrm{~Hz}$ and the time range from $-5.0 \mathrm{~s}$ to $5.0 \mathrm{~s}$, i.e. with 1501 time steps. The response has been evaluated in frequency domain by application of the semi-analytical model described in Sections 2.2-2.3 for the 301 discrete frequencies up to $30 \mathrm{~Hz}$, and zero padding has been applied before inverse Fourier transformation 
back to time domain. Figure 19 contains five snapshots of the ground surface at the times 0.1 , $0.2,0.4,0.8$ and $1.6 \mathrm{~s}$, clearly showing the wave front of the surface wave propagating along the surface. Especially, at the instants 0.4 and $0.8 \mathrm{~s}$, the wave pattern becomes more complex due to reflections of waves at the layer interface and scattering by the WIBs.

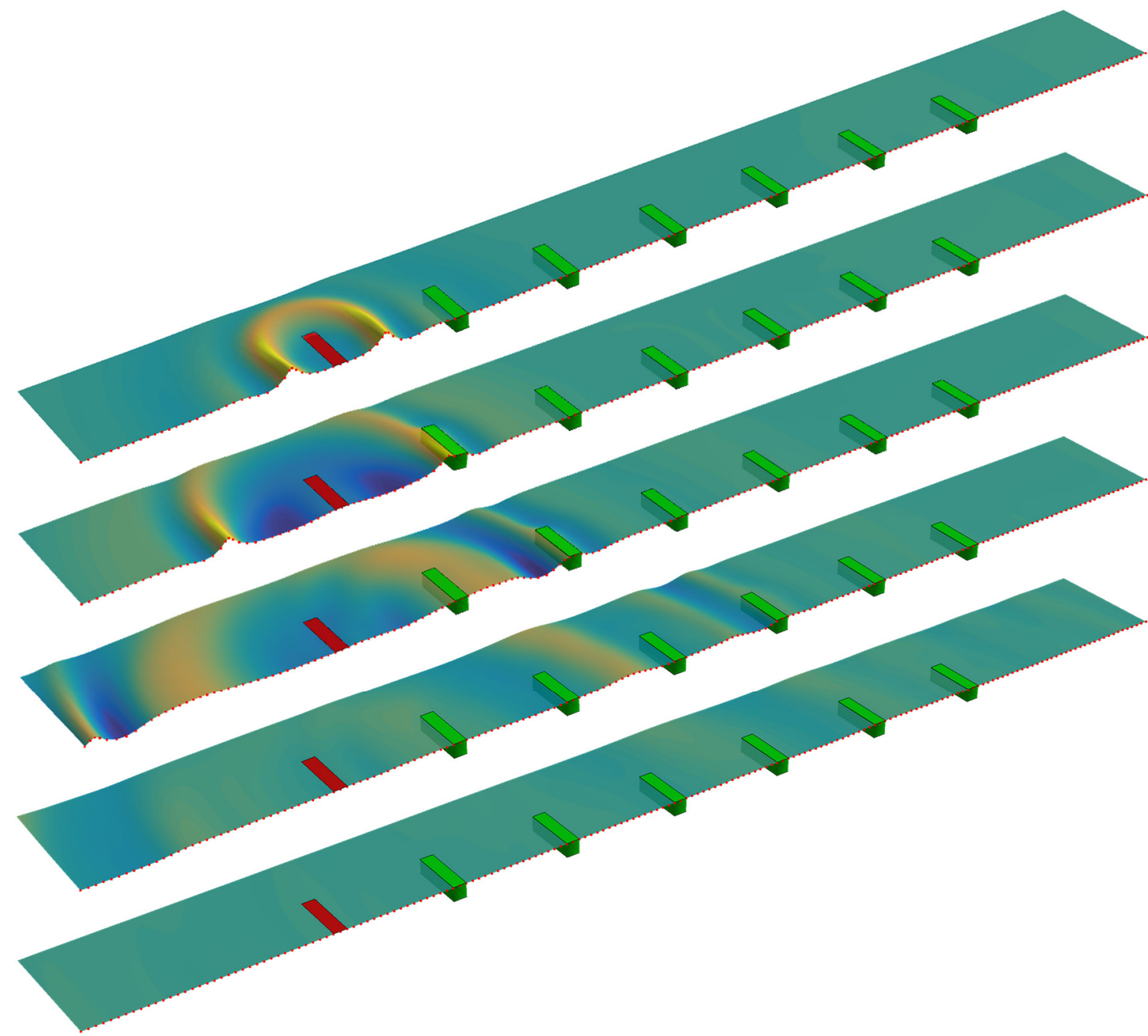

Figure 19: Response of ground surface and array with six (green) WIBs to a Ricker wavelet applied with centre frequency $10 \mathrm{~Hz}$ and centre time $0 \mathrm{~s}$ on the rigid (red) plate. The snapshots are shown (from top to bottom) for the times $0.1,0.2,0.4,0.8$ and $1.6 \mathrm{~s}$, i.e. after $1,2,4,8$ and 16 periods associated with the centre frequency.

Figure 20 shows the time histories of the Ricker wavelet and the resulting vertical groundsurface displacements for a reference case with no WIBs embedded in the ground (only soil). The displacement have all been normalized by the reference displacement $100 \mathrm{pm}$. This means that the actual displacement that would occur for a pulse with magnitude $10 \mathrm{kN}$ would be about $1 \mu \mathrm{m}$ at a distance of $45 \mathrm{~m}$ away from the loaded rigid plate. The delay in first arrivals at the three positions $x=45 \mathrm{~m}, x=81 \mathrm{~m}$ and $x=117 \mathrm{~m}$ can be seen clearly. Further, the first wave to arrive travels via the stiffer sublayer and has significantly smaller amplitude than the next wave which propagates along the surface of the softer layer. An interesting observation can be made regarding the response at the position $x=117 \mathrm{~m}$. Here the vibration decays slowly with time and the period of the vibrations is approximately $0.21 \mathrm{~s}$, i.e. about twice the centre frequency of the Ricker wavelet. This indicates that resonance in the stratum rather than the exact shape of the load signal dominates the behaviour at large distances from the load. 


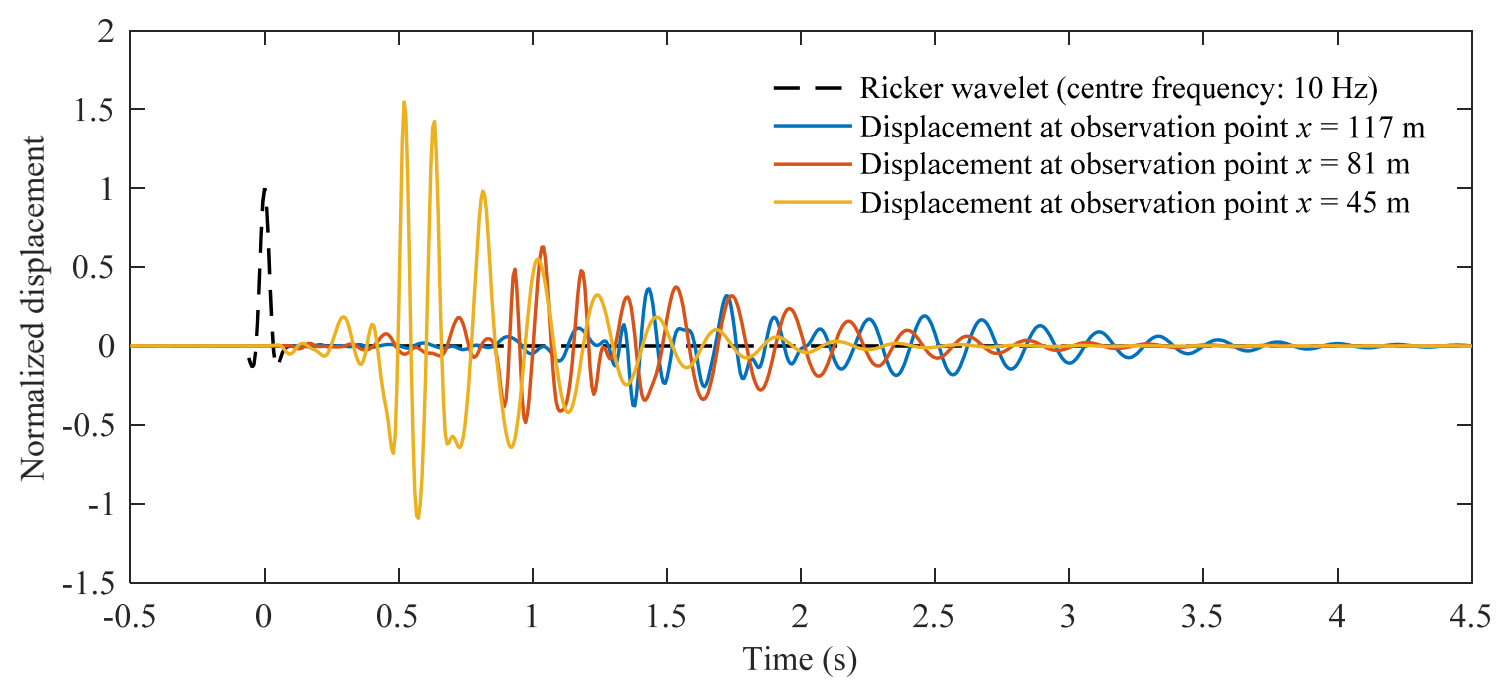

Figure 20: Ricker wavelet applied at $x=0 \mathrm{~m}$ with centre frequency $10 \mathrm{~Hz}$ and displacements at three observation points, normalized with respect to a reference displacement of $100 \mathrm{pm}$, for reference case with no wave impeding blocks placed in the ground.

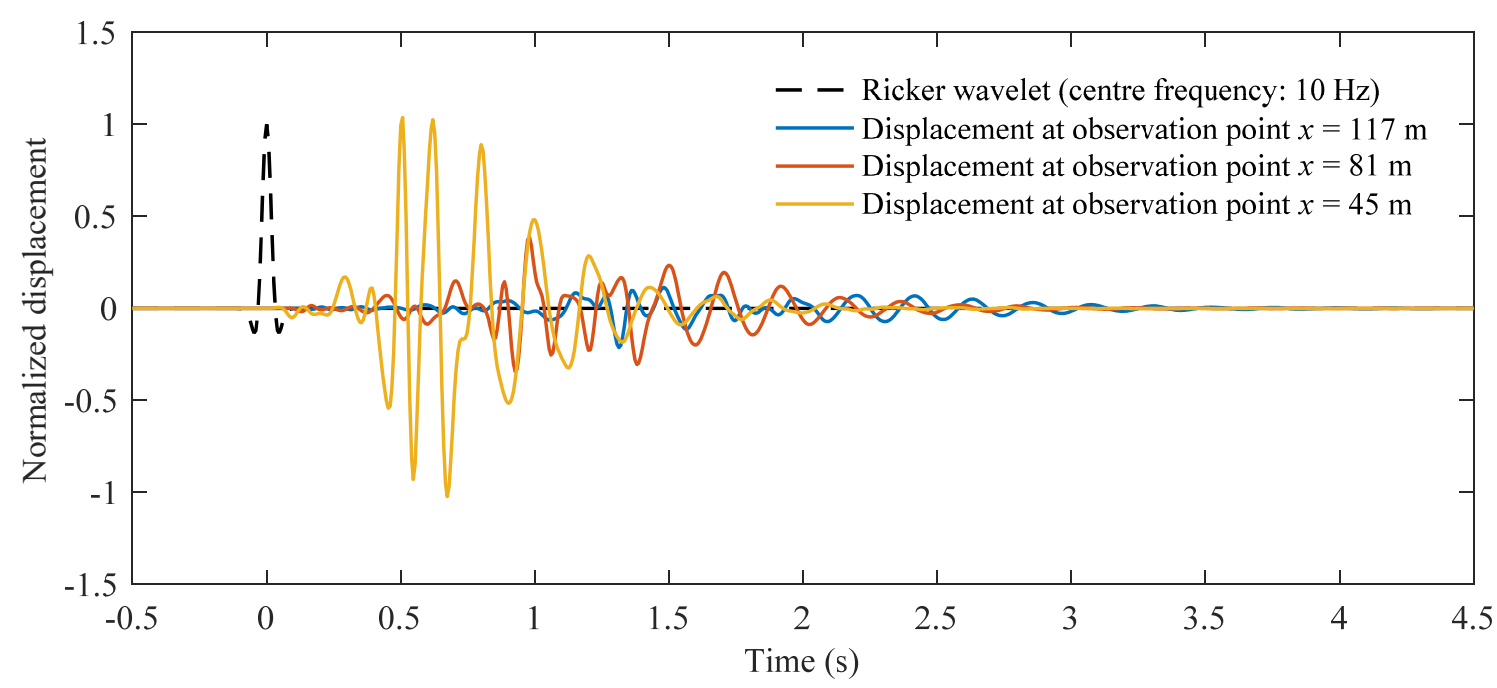

Figure 21: Ricker wavelet applied at $x=0 \mathrm{~m}$ with centre frequency $10 \mathrm{~Hz}$ and displacements at three observation points, normalized with respect to a reference displacement of $100 \mathrm{pm}$, for case with an array of six wave impeding blocks embedded $2 \mathrm{~m}$ into the ground.

Next, Figure 21 shows the Ricker wavelet and the resulting vertical ground-surface displacements for the case with six WIBs embedded in the ground, i.e. the case illustrated in Figure 19. Compared to the displacement response in the reference case without WIBs (see Figure 20), smaller vibration levels are observed-especially at the position $x=117 \mathrm{~m}$ beyond the WIB array. Figure 22 illustrates the response at this position for different numbers of WIBs in the array including the reference case (only soil) with no blocks. Again, the Ricker wavelet has been included to visualize the delay in time between application of the load and first arrival of the waves. The effect of the WIB array is clear and the vibration response becomes smaller with every addition of more blocks. This can as well be observed in Figure 23, showing the Fourier spectra of the Ricker wavelet and the four time-series of vertical displacements at $x=117 \mathrm{~m}$. Especially, great reduction occurs in the frequency range from $4-8 \mathrm{~Hz}$ and around $13 \mathrm{~Hz}$, matching well with the observation made regarding the IL related to the steady state. Further, the spectrum for the Ricker wavelet shows that the excitation contains no significant energy at 
frequencies above $25 \mathrm{~Hz}$ (note that the tail of the spectrum decreases monotonically). This justifies the calculation procedure in which the response has only been calculated by the semianalytical model for frequencies up to $30 \mathrm{~Hz}$.

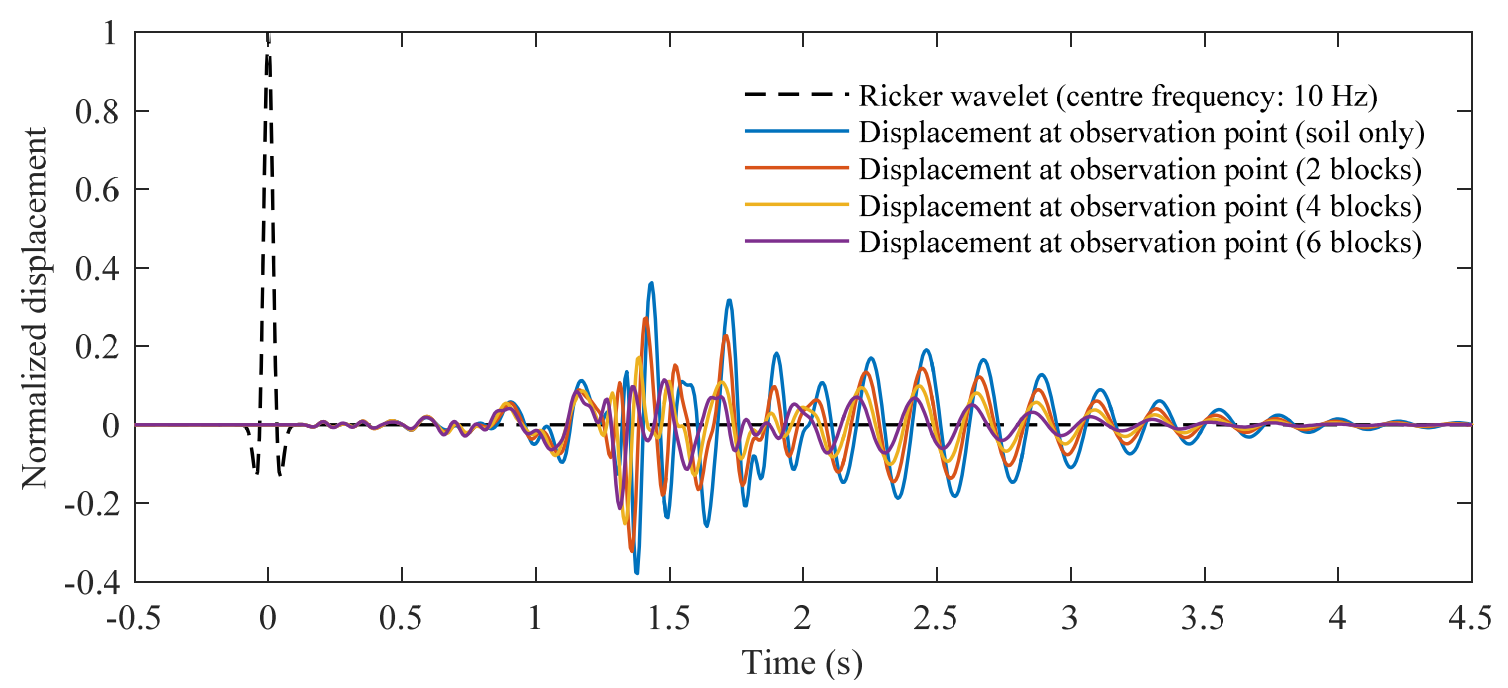

Figure 22: Ricker wavelet applied at $x=0 \mathrm{~m}$ with centre frequency $10 \mathrm{~Hz}$ and displacements at $x=117 \mathrm{~m}$, normalized with respect to a reference displacement of $100 \mathrm{pm}$.

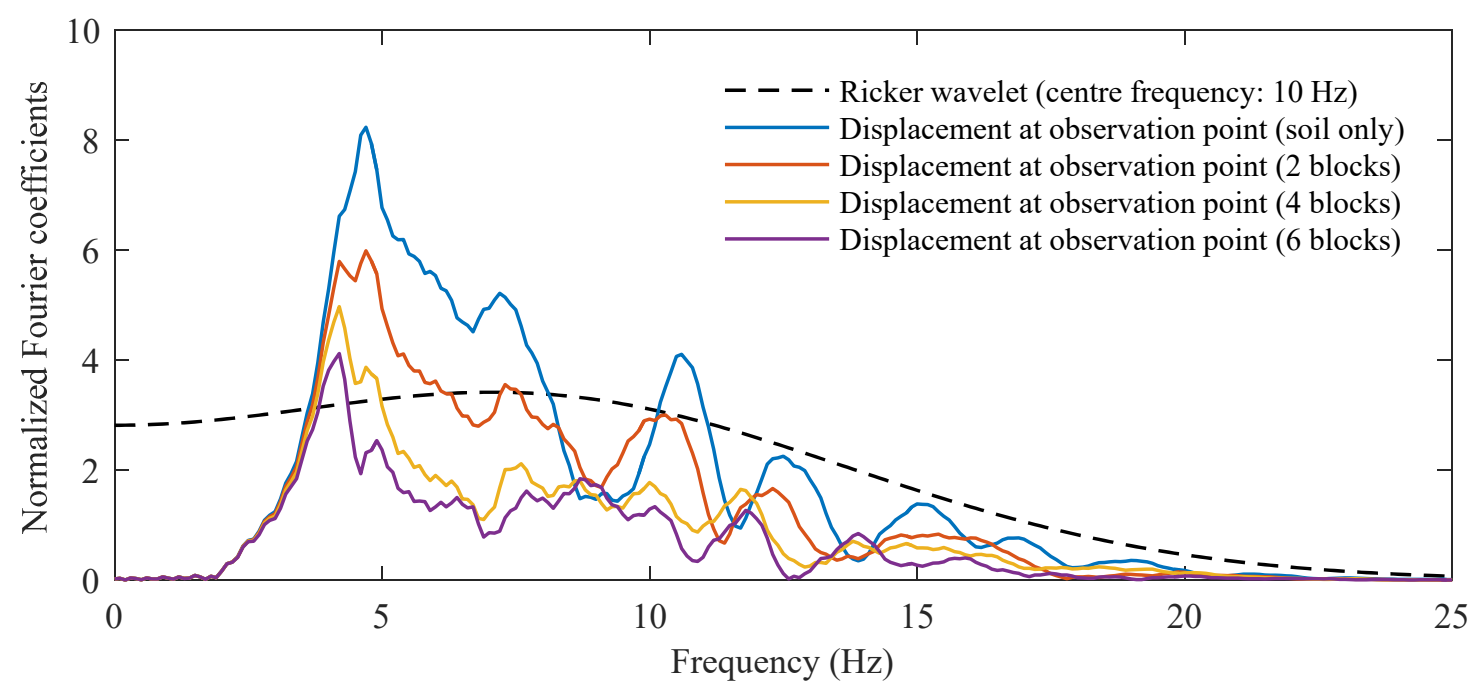

Figure 23: Fourier coefficients of a unit Ricker wavelet applied at $x=0 \mathrm{~m}$ with centre frequency $10 \mathrm{~Hz}$, normalized with respect to a reference load of $1 \mathrm{mN}$, and Fourier coefficients of displacements at $x=117 \mathrm{~m}$, normalized with respect to a reference displacement of $0.1 \mathrm{pm}$.

\section{CONCLUSIONS}

Wave impeding blocks (WIBs) have been proposed as a method of reducing low-frequency ground vibration. A model, which uses the Green's function transfer-matrix technique, has been implemented for three-dimensional analysis of WIB arrays placed on the surface of or embedded within two-layered strata. The model consisted of a $10 \mathrm{~m}$ deep sand layer over a $20 \mathrm{~m}$ deep till layer on bedrock, and the WIBs were modelled as rigid blocks. To characterise the onset of modal wave propagation, a 2D finite-element model was adopted from ABAQUS. Employing Floquet periodicity conditions to a reference cell, allowed us to analyse the behaviour of an infinite structure including a periodic array of WIBs situated within the upper stratum. 
Various configurations of finite WIB arrays have been analysed in 3D. The 2D model has been used for comparison to identify whether vibration mitigation occurs in the 3D model within the frequency ranges where the infinite periodic WIB array produced stopbands. Hence, the article has presented a number of characteristics related to WIBs in periodic and nearly periodic arrays embedded within or placed on the surface of stratified media:

- A WIB can be used to affect the wave-propagation behaviour of layered media significantly. A "source-free" two-dimensional analysis verifies the existence of stopbands in which no propagating wave modes occur.

- The frequency range that can be controlled by this technique is low enough to be useful for the control of the vibration phenomenon at low frequency associated with surface-running freight trains, for example.

- The phenomenon of stopband behaviour is particularly useful for designing a "vibration mitigation device" consisting of a periodic linear array of masses. Reductions up to $30 \mathrm{~dB}$ were observed when the WIBs were placed just under the ground surface.

- It has been shown that periodic arrays of WIBs submerged just under the ground surface exhibit consistently high mitigation capabilities. Smaller blocks sitting at the ground surface may produce negative insertion loss in the low-frequency range, and submerging the blocks further into the ground produces lower insertion loss at higher frequencies.

- The importance of having equidistantly placed WIBs to produce a finite periodic structure was examined. Somewhat surprisingly, slight variations in individual positions of WIBs in an array could be advantageous over a broad frequency range, compared to an "exact" periodic array. Moreover, it was found that the large insertion losses exhibited by an exact periodic array is not lost by slight variations of block locations. Up to variations to one block width, the advantageous insertion loss due to the array were still evident.

- Time-domain solutions are essential in providing true evidence of mitigation-especially if modelling short-term vehicle pass-bys from trucks or trains. The semi-analytical model proves to be a versatile method since the model produces time-domain solutions at receivers in relatively short time. The results prove that for very short-term time intervals, in the mid-term, the vibration response can increase at receiver positions. This effect is shortlived but dominated by a general monotonic reduction in displacement as the number of blocks increases.

Further analysis includes:

- A suitable source-free methodology to describe a "reduction" in energy propagation in a half-space for both 2D and 3D problems not unlike the Floquet periodicity principle.

- A 3D analysis for the relative number of propagating wave modes in layered media.

- Optimisation of the positions of individual WIBs in non-periodic arrays to maximize the insertion loss in a broad frequency range.

\section{ACKNOWLEDGMENTS}

The research was carried out in the framework of the project "Urban Tranquility" under the Interreg V programme. The authors of this work gratefully acknowledge the European Regional Development Fund for the financial support. 


\section{REFERENCES}

[1] L. V. Andersen, "Using periodicity to mitigate ground vibration," in COMPDYN 2015 5th ECCOMAS Thematic Conference on Computational Methods in Structural Dynamics and Earthquake Engineering, 2015.

[2] L. V. Andersen, P. Bucinskas, P. Persson, M. Muresan, L.-I. Muresan, and I.-O. Paven, "Mitigating ground vibration by periodic inclusions and surface structures," in Proceedings of the INTER-NOISE 2016 - 45th International Congress and Exposition on Noise Control Engineering: Towards a Quieter Future, 2016.

[3] G. B. Warburton, H. D. Richardson, and J. J. Webster, "Harmonic response of masses on an elastic half-space," J. Eng. Ind. Trans. ASME, vol. 75, pp. 158-170, 1972.

[4] V. V. Krylov, "Scattering of Rayleigh waves by heavy masses as method of protection against traffic-induced ground vibrations," in Environmental Vibrations: Prediction, Monitoring, Mitigation and Evaluation (ISEV 2005), 2005, pp. 393-398.

[5] A. Dijckmans, P. Coulier, J. Jiang, M. G. R. Toward, D. J. Thompson, G. Degrande, G. Lombaert, "Mitigation of railway induced ground vibration by heavy masses next to the track," Soil Dyn. Earthq. Eng., vol. 75, pp. 158-170, 2015.

[6] A. T. Peplow, C. J. C. Jones, and M. Petyt, "Surface vibration propagation over a layered half-space with an inclusion," Appl. Acoust., vol. 56, pp. 283-296, 1999.

[7] A. T. Peplow and S. Finnveden, "Calculation of vibration transmission over bedrock using a waveguide finite element model," Int. J. Numer. Anal. methods Geomech., vol. 32, no. 6, pp. 701-719, 2008.

[8] H. Takemiya, "Field vibration mitigation by honeycomb WIB for pile foundations of a high-speed train viaduct," Soil Dyn. Earthq. Eng., vol. 24, no. 1, pp. 69-87, 2004.

[9] H. Masoumi, A. Van Leuven, and S. Urbaniak, "Mitigation of train induced vibrations by wave impeding blocks: numerical prediction and experimental validation," in EURODYN 2014, 2014, no. July, pp. 863-870.

[10] L. Andersen and S. R. K. Nielsen, "Reduction of ground vibration by means of barriers or soil improvement along a railway track," Soil Dyn. Earthq. Eng., vol. 25, no. 7-10, pp. 701-716, Aug. 2005.

[11] L. Andersen and M. Liingaard, "Vibration screening with sheet pile walls," in Environmental Vibrations: Prediction, Monitoring, Mitigation and Evaluation (ISEV 2005), 2005, pp. 429-437.

[12] L. Andersen and A. H. Augustesen, "Mitigation of traffic-induced ground vibration by inclined wave barriers - A three-dimensional numerical analysis," in 16th International Congress on Sound and Vibration 2009, ICSV 2009, 2009, vol. 4.

[13] C. van Hoorickx, O. Sigmund, M. Schevenels, B. S. Lazarov, and G. Lombaert, "Topology optimization of two-dimensional elastic wave barriers," J. Sound Vib., vol. 376, pp. 95-111, 2016.

[14] D. J. Mead, "Free wave propagation in periodically supported, infinite beams," J. Sound Vib., vol. 11, no. 2, pp. 181-197, 1970.

[15] P. Persson, K. Persson, and G. Sandberg, "Reduction in ground vibrations by using shaped landscapes," Soil Dyn. Earthq. Eng., vol. 60, pp. 31-43, 2014. 
[16] Dassault Systèmes Simulia Corp., “Abaqus 6.14.” Dassault Systèmes Simulia Corp., Providence, RI, USA.

[17] J. Lysmer and R. L. Kuhlemeyer, "Finite Dynamic Model for Infinite Media," J. Eng. Mech. Div. Proc. ASCE, vol. 4, pp. 859-877, 1969.

[18] W. C. Chew and Q. H. Liu, "Perfectly matched layers for elastodynamics: A new absorbing boundary condition," J. Comput. Acoust., vol. 4, no. 4, pp. 341-359, Dec. 1996.

[19] N. A. Haskell, "The dispersion of surface waves on multilayered media," Bull. Seismol. Soc. Am., vol. 43, no. 1, pp. 17-34, 1953.

[20] W. T. Thomson, "Transmission of Elastic Waves through a Stratified Solid Medium," J. Appl. Phys., vol. 21, no. 2, pp. 89-93, Feb. 1950.

[21] X. Sheng, C. J. C. Jones, and M. PETYT, "Ground vibration generated by a load moving along a railway track," J. Sound Vib., vol. 228, no. 1, pp. 129-156, Nov. 1999.

[22] L. Andersen and J. Clausen, "Impedance of surface footings on layered ground," Comput. Struct., vol. 86, no. 1-2, pp. 72-87, 2008.

[23] L. V. Andersen, "Dynamic soil-structure interaction of monopod and polypod foundations," in Insights and Innovations in Structural Engineering, Mechanics and Computation, Proceedings of the Sixth International Conference on Structural Engineering, Mechanics and Computation, 5-7 September 2016, Cape Town, South Africa, 2016, pp. 2036-2041.

[24] P. Bucinskas, L. V. Andersen, and K. Persson, "Numerical modelling of ground vibration caused by elevated high-speed railway lines considering structure-soil-structure interaction," in Proceedings of the INTER-NOISE 2016 - 45th International Congress and Exposition on Noise Control Engineering: Towards a Quieter Future, 2016. 